\title{
DISCLAIMER
}

This report was prepared as an account of work sponsored by an agency of the United States Government. Neither the United States Government nor any agency thereof, nor any of their employees, makes any warranty, express or implied, or assumes any legal liability or responsibility for the accuracy, completeness, or usefulness of any information, apparatus, product, or process disclosed, or represents that its use would not infringe privately owned rights. Reference herein to any specific commercial product, process, or service by trade name, trademark. manufacturer, or otherwise does not necessarily constitute or imply its endorsement, recommendation, or favoring by the United States Government or any agency thereof. The views and opinions of authors expressed herein do not necessarily state or reflect those of the and opinions of authors expressed herein do
United States Government or any agency thereof.

\section{Investigation of Pion-Nucleus Interactions}

\author{
Progress Report
}

April 1, 1990 - March 31, 1991

C. Fred Moore

Department of Physics

University of Texas at Austin

Austin, Texas 78712-1081

September 1991

\section{PREPARED FOR THE U. S. DEPARTMENT OF ENERGY UNDER GRANT NUMBER DE-FG05-87ER 40343}

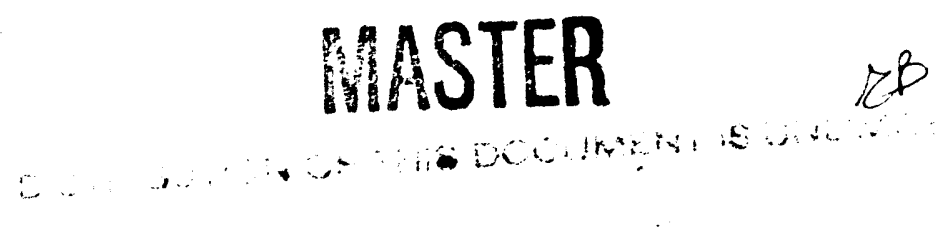




\section{Contents}

1 Introduction $\quad 2$

2 Overview $\quad 3$

3 The double giant dipole resonance in the $\left(\pi^{-}, \pi^{+}\right)$reaction 5

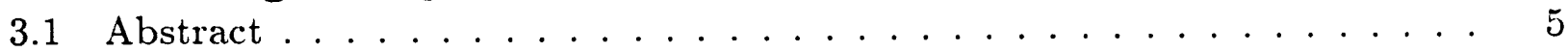

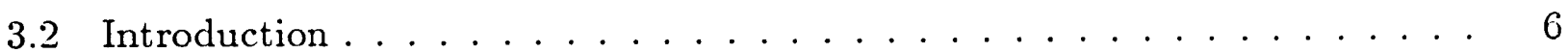

3.3 Results and Analysis . . . . . . . . . . . . . . . . 6

3.3 .1 Discussion of the data . . . . . . . . . . . . 6

3.3.2 Energy relations and symmetry energy . . . . . . . . . . . . 9

3.3.3 Angular distribution of the double dipole . . . . . . . . . . . . 13

3.3.4 Width of the double-dipole resonance . . . . . . . . . . . . 13

3.3.5 Cross-section ratios and background analysis . . . . . . . . . . 16

3.3.6 Mass dependence of the double dipole . . . . . . . . . . . 22

4 Publications $\quad 28$

5 Abstracts $\quad 31$

6 Invited Talks $\quad 32$

7 Proposal Defenses $\quad 32$

8 Approved New Research Proposals $\quad 33$

9 Personnel $\quad 34$

\section{List of Tables}

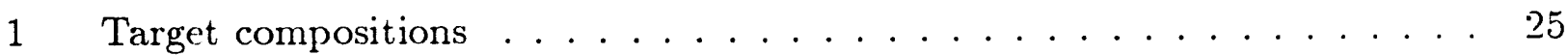

2 Double dipole $Q$ values and excitation energies . . . . . . . . . . 25

$3 \quad\left(\pi^{+}, \pi^{-}\right)$and $\left(\pi^{-}, \pi^{+}\right) \ldots \ldots \ldots \ldots \ldots$

4 Comparison between DCX SCX and theoretical estimates . . . . . . . . 27

\section{List of Figures}

1 Doubly differential cross-section spectrum .............. . 7

2 Doubly differential cross-section spectrum ............. . \&

3 Level scheme for DCX to the double isovector giant dipole resonances . . . 10

$4 Q$-values versus $A \ldots \ldots \ldots \ldots \ldots$

5 Angular distribution for double di ${ }_{\ell}$ oles . . . . . . . . . . . . 14 
Widths of the double isovector giant dipole state . . . . . . . 15

7 Ratio of the double dipole cross sections . . . . . . . . . . . . . . 17

8 Fitted background spectra . . . . . . . . . . . . . . . . . 19

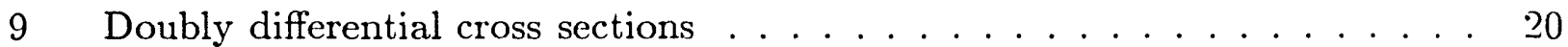

10 Both DCX mode spectra for ${ }^{40} \mathrm{Ca} \ldots \ldots \ldots \ldots \ldots \ldots . \ldots \ldots$

11 Plots of the double dipole cross sections $\ldots \ldots \ldots \ldots$

\section{Introduction}

This report summarizes the work carried out by personnel from the University of Texas at Austin at the Los Alamos Clinton P. Anderson Meson Physics Facility (LAMPF) during. the calendar years 1990-1991 under grant DE-FG05-87ER40343 between the University of Texas at Austin and the United States Department of Energy.

One of our faculty and a research faculty member has been on site (at LAMPF) during five months or more of each year. An average of eight graduate students have been associated with this program, most being in residence at LAMPF.

The research activities involved experiments done with the Energetic Pion Channel and Spectrometer (EPICS), the Low Energy Pion Channel (LEP), the Pion and Particle Physics Channel $\left(\mathrm{P}^{3}\right)$, and the High Resolution Spectrometer (HRS).

A brief overview of work supported by this grant is given in Section 2. An account of the study (Experiment \#1150) of the double giant dipole resonance with the $\left(\pi^{-}, \pi^{+}\right)$reaction forms Section 3. This report contains a list of published papers and preprints in Section 4, abstracts in Section 5, and invited talks in Section 6. These papers summarize experiments involving participants supported by this grant and indicate the work accomplished by these participants in this program of medium energy nuclear physics research. Section $\mathbf{7}$ contains a list of the most recent proposals on which we have participation at LAMPF, while Section 8 lists proposals which have been approved this past year to run as experiments. Section 9 contains a list of personnel who have participated in this research program.

The research cited in this report is, in many cases, the collaborative effort of many groups associated with research at LAMPF. Collaboration with the Los Alamos National Laboratory, the University of Minnesota, the University of Colorado, the Louisiana State University, the Ben Gurion University of the Negev, the King Fahd University of Petroleum and Minerals, the Lawrence Livermore Laboratory, the New Mexico State University, Argonne National Laboratory, the University of Colorado, and the University of Pennsyluania is acknowledged.

One Ph.D. degree was granted by the University of Texas at Austin during 1991, based on experimental research done at LAMPF, bringing the total number of Ph.D.'s granted to Texas students supported by this grant in meson-nuclear physics to twelve. 


\section{Overview}

Our program concentrates on pion physics experimental results obtained using the Encrgetic Pion Channel and Spectrometer (EPICS), Pion and Particle Physics channcl ( $P^{3}$ ), and the Low Energy Pion physics channel (LEP). These facilities are unique in the world in their intensity and resolution. Two classes of experiments can be done best with this equipment: scattering (elastic and inelastic) and doubie charge exchange (DCX). Sevcral coincidence experiments are in progress and several are proposed.

Inelastic pion scattering proposal \#1025, which has been approved with $\mathrm{A}^{+}$priority, deals with measurements of pion scattering from a polarized ${ }^{13} \mathrm{C}$ target. The asymmetry parameter $\mathrm{A}_{y}$ for $\pi^{ \pm}$elastic and inelastic scattering has been measured over an angular range of $30^{\circ}$ to $100^{\circ}$ from a polarized ${ }^{13} \mathrm{C}$ target at EPICS. The elastic-scattering results should be sensitive to spin-dependent effects in the pion-nucleus interaction, such as the $\Delta$-nucleus spin-orbit interaction. The inelastic-scattering results will be sensitive to the relative mixture of spin-flip and non-spin-flip contributions to the transitions. These polarized target experiments will give new information about the pion-nucleus interaction that is not available from experiments with unpolarized targets, because the former moasures the interference between amplitudes and the latter measures squares of amplitudes. The target system was assembled principally from polarized-target elements available at LAMPF. The experimental beam time requested is one running cycle, taken to be 1,200 hours.

In experiment \#1104 cross-sections to the GQR for the nuclei ${ }^{90} \mathrm{Zr}$ and ${ }^{208} \mathrm{~Pb}$ have been measured with $\pi^{+}$and $\pi^{-}$at energies from 350 to $550 \mathrm{MeV}$, i.e., above the $\Delta_{3 / 2,3 / 2}$ resonance region. The goal of this experiment is more accurate evaluation of neutron and proton transition amplitudes $\left(M_{n}\right.$ and $\left.M_{p}\right)$ extracted for GQR in those neutron excess nuclei. Those values of $M_{n}$ and $M_{p}$ obtained near the $\Delta_{3 / 2,3 / 2}$ resonance region have uncertainties in the wave functions involved and the shapes of the neutron and proton transition densities at the nuclear surface due to the short mean free path of the pion near the $\Delta$ resonance. The longer mean free path of the pion in these higher energy region may help to eliminate those uncertainties in extraction of the matrix elements.

Regarding proposal \#1018, we have analyzed large-angle pion elastic data on ${ }^{12} \mathrm{C}$ and ${ }^{16} \mathrm{O}$ with standard first-order optical models using matter distributions deduced from electron scattering. We show that these models reproduce only the qualitative features of the data. However, much better agreement can be achieved with the same models provided the matter distributions are modified in a suitable way. At this stage it is not clear whether these corrections are applicable to all nuclei or whether they apply at energies other than those on resonance. To address these questions we propose to measure clastic excitation functions (at $175^{\circ}$ ) on ${ }^{6} \mathrm{Li},{ }^{28} \mathrm{Si}$, and ${ }^{58} \mathrm{Ni}$ in the energy range from 100 to $250 \mathrm{McV}$.

Pion absorption: The LEP and $\mathrm{P}^{3}$ channels at LAMPF will be used. In the low energy pion channel, we propose to measure the cross sections for $\pi^{+}$and $\pi^{-}$absorption on ${ }^{12} \mathrm{C}$, ${ }^{58} \mathrm{Ni}$, and ${ }^{208} \mathrm{~Pb}$ in which the final state includes two or more free protons. Data will be taken for $\pi$ energies of 50,100,150, and $200 \mathrm{MeV}$. The energy and angular distributions 
of the outgoing protons are measured using a large solid-angle bismuth germanate BGO detector described in the section of this proposal entitled "BGO BALL".

In the pion particle physics $\left(\mathrm{P}^{3}\right)$ channel, we measure the cross sections for $\pi^{+}$and $\pi^{-}$absorption on ${ }^{12} \mathrm{C},{ }^{58} \mathrm{Ni}$, and ${ }^{208} \mathrm{~Pb}$ in which the final state includes two or more free protons. Data are taken for $\pi$ energies of $200,300,400$, and $500 \mathrm{MeV}$. The energy and angular distributions of the outgoing protons are measured using a large solid-angle BGO detector.

Among the most exciting results obtained recently at LAMPF in pion physics is the observation of resolved giant-dipole resonances built on the isobaric analog states and the isovector giant dipole resonance built on another giant dipole. The isobaric analog, and the giant dipole were both observed in $\left(\pi^{+}, \pi^{-}\right)$single charge exchange reaction (with a typical maximum cross section around $0.5 \rightarrow 1.0 \mathrm{mb} / \mathrm{sr}$ ). Therefore a combination of these resonances could, in principal, be reached in double charge exchange. However, until recently, none of these double resonances had been found.

The results obtained so far are exciting and have a potential of new and interesting physics. Experiments \#1050 and \#1118 have demonstrated that pion DCX provide a unique reaction in which one can study the existence of double giant resonances in nuclei. The results indicate that two new different modes of nuclear excitations are observable in pion double charge exchange reactions. These are the giant dipole built on the isobaric analog state and the giant dipole resonance built on the giant dipole resonance. Due to the selectivity of the DCX reaction these exotic states are observable although they are located at high excitation energy where background from DCX to the continuum is large. The data show that the newly discovered resonances are general collective features of all nuclei for the double-dipole, and of all nuclei with $N-Z \geq 1$ for the GDR built on the IAS. In experiment \#1118 at the energetic pion channel and spectrometer (EPICS) at LAMPF, we observed a clear splitting of the giant dipole built on the isobaric analog state (GDR $\otimes I A S$ ) in the ${ }^{93} \mathrm{Nb}\left(\pi^{+}, \pi^{-}\right){ }^{93} \mathrm{Tc}$ reaction at $\mathrm{T}_{\pi}=295 \mathrm{MeV}$. The results obtained i:s the first two research years of this program have been published (or submitted) in several scientific reports and invited talks at international conferences.

Experiment \#1154 (which ran in two phases) was designed to accurately measure the width of the giant dipole built on the isobaric analog state on a wide range of mass and with higher statistics. The results indicate that the width of the GDR built on the IAS increases significantly with mass. For example, the resonance for ${ }^{13} \mathrm{C}$ has a width of $3.0 \pm 0.6$ $\mathrm{MeV}$, but that for ${ }^{59} \mathrm{Co}$ has a width of $7.0 \pm 1.0 \mathrm{MeV}$ (more than twice as wide as for ${ }^{13} \mathrm{C}$ ). The increase of the width with mass is not yet fully understood, and is one of the puzzles arising from this study. An interesting implication was also discovered during this study. The resonance on the $T=1 / 2$ targets has a single isospin value $(T=3 / 2)$ and is the analog of the $T_{>}$GDR state in the target nucleus. Thus pion DCX provides a new general method for an accurate devermination of the $T_{>}=T+1$ giant dipole state on any $T=1 / 2$ nucleus available as a target for DCX measurements.

Multiparticle final state in pion interactions: We studied the study of decay protons in the reaction $\left(\pi, \pi^{\prime} p\right)$ as a test of charge symmetry. Comparisons of photo proton and photo neutron breakup of ${ }^{4} \mathrm{He}$ have provided evidence for a large violation of charge symmetry, 
caused by forces other than the Coulomb force. However, pion inelastic scattering data show that the deviation from charge symmetry can be explained by the Coulomb potential only. In an attempt to resolve this argument on the existence of charge symmetry breaking forces other than Coulomb interaction, a series of $\left(\pi, \pi^{\prime} \mathrm{p}\right)$ coincidence experiments on ${ }^{4} \mathrm{He}$ (\#998), ${ }^{12} \mathrm{C}$ and ${ }^{208} \mathrm{~Pb}$ (\#1039) were conducted during 1986-91 time period. The conclusion of these experiments is that there is no other force than Coulomb that breaks up charge symmetry, and the discrepancy between the result using electromagnetic probes and that using pions can be explained by direct-knock-out and semi-direct components, using the widely used Direct-Semidirect Model or Doorway-state Model.

Experiment \#1103 aims to measure the ratio of $\left(\pi^{+}, \pi^{+\prime}\right)$ vs $\left(\pi^{-}, \pi^{-\prime}\right)$ on various nuclei to provide information on proton and neutron transition densities at energies around the Roper resonance where the relative strength of pure proton and pure neutron transitions should be reversed as compared to the $\Delta_{3 / 2,3 / 2}(1232)$ resonance case.

Experiment \#1210 ran very recently at LEP (July 1991). The experiment aimed to study the mechanism for DCX to excite analogs of anti-analog states in heavy nuclei. A previous, high statistics DCX experiment on ${ }^{93} \mathrm{Nb}$ showed for the first time the existcnce of a resonance at high excitation energy below the double isobaric analog state. The observation of the resonance with such a large cross section was unexpected. In a recent article we suggested a few possible identifications for this resonance. Experiment \#1210 confirms the previous observation on ${ }^{93} \mathrm{Nb}$ and gives new results on ${ }^{56} \mathrm{Fe}$ and ${ }^{44} \mathrm{Ca}$ at a lower beam energy. The results are still under study, and additional beam time was approved for this experiment by the PAC.

In Section 3 we discuss in detail the results from Experiment \#1150 which ran at EPICS in Sep-Oct 1990.

\section{The double giant dipole resonance in the $\left(\pi^{-}, \pi^{+}\right)$ reaction}

\subsection{Abstract}

The double isovector giant dipole state has been observed in the $\left(\pi^{-}, \pi^{+}\right) \Delta T_{z}=+2$ double-charge-exchange reaction on ${ }^{13} \mathrm{C},{ }^{27} \mathrm{Al},{ }^{40} \mathrm{Ca},{ }^{56} \mathrm{Fe},{ }^{59} \mathrm{Co}$, and ${ }^{93} \mathrm{Nb}$. The resonances observed in the $\left(\pi^{-}, \pi^{+}\right)$reaction are closely related via Coulomb displacement cuergy and isospin symmetry to the resonances measured in the inverse $\left(\pi^{+}, \pi^{-}\right)$reaction on the same nuclei. The new observations provide a direct determination of the upper isospin component of the double giant dipole state, which is generally very weak in the $\left(\pi^{+}, \pi^{-}\right)$ reaction. The comparison between the double dipole in the two double-charge-excliangc modes gives valuable information on the isospin splitting and the Pauli-blocking effects for isotensor transitions. 


\subsection{Introduction}

In a recent paper[1] we reported the first observation of a double isovector giant dipole resonance in nuclei in the $\left(\pi^{+}, \pi^{-}\right)$double-charge-exchange (DCX) reaction on ${ }^{\text {nat }} \mathrm{S}$. More recent data[2,3] clearly show the existence of these exotic resonances in the continum at high excitation energies. The identification of the double-dipole resonances is based on their centroid energies, characteristic angular distributions, cross sections, and comparison with the inverse DCX reaction[2,3]. Pion DCX offers a cleaner way to study double giant resonances than that offered by inelastic reactions because of the selectivity of the DCX reaction and the clear geometrical signature of the angular distributions of the various giant resonances (GR) due to the strong absorption of the pions. An additional unique feature of pion DCX used in this study is the simplicity with which one can study the inverse reaction. There are four advantages to measuring the double-dipole resonance in the $\left(\pi^{-}, \pi^{+}\right)$reaction: a) The double dipole is expected to appear at a significantly lower energy and with a lower background level from the continuum than in the $\left(\pi^{+}, \pi^{-}\right)$mode. The decrease in energy of the double dipole in the $\left(\pi^{-}, \pi^{+}\right)$reaction is about four Coulomb energies (after correcting for the symmetry energy and the neutron-proton mass difference), and that is a significant shift for medium and heavy nuclei. For example, the double clipole is observed at $Q=-49.9 \mathrm{MeV}\left(E_{x}=47.3 \mathrm{MeV}\right.$ in ${ }^{93} \mathrm{Tc}$ ) in ${ }^{93} \mathrm{Nb}\left(\pi^{+}, \pi^{-}\right){ }^{93} \mathrm{Tc}$, but (as we see below) in the inverse reaction it appears at $Q=-24.9 \mathrm{MeV}\left(E_{x}=20.9 \mathrm{MeV}\right.$ in $\left.{ }^{93} \mathrm{Y}\right)$. b) In $\left(\pi^{-}, \pi^{+}\right)$the spectra should be simpler because the double isobaric analog state (DIAS) and the giant dipole built on the isobaric analog state (GDR $\otimes I A S$ ) resonances do not exist, and therefore the double dipole is the lowest resonance expected in the rcaction. c) The double dipole is expected to have a smaller width in $\left(\pi^{-}, \pi^{+}\right)$than in the inverse reaction. d) The double GDR reached in $\left(\pi^{-}, \pi^{+}\right)$has a single isospin component, as will be discussed later. However, we note that measurements of $\left(\pi^{-}, \pi^{+}\right)$are much more difficult than $\left(\pi^{+}, \pi^{-}\right)$, because $\pi^{+}$beam fluxes are generally larger by about a factor of six relative to the $\pi^{-}$fluxes at the energies of interest for the present study. Furthermore, for all $N>Z$ nuclei the cross sections for the double dipole in $\left(\pi^{-}, \pi^{+}\right)$are smaller than those in $\left(\pi^{+}, \pi^{-}\right)$because of the Pauli-blocking effect, making the running time for a single measurement significantly longer.

\subsection{Results and Analysis}

\subsubsection{Discussion of the data}

Figure 1 presents the ${ }^{13} \mathrm{C}\left(\pi^{-}, \pi^{+}\right){ }^{13} \mathrm{Be}$ and the previous ${ }^{13} \mathrm{C}\left(\pi^{+}, \pi^{-}\right){ }^{13} \mathrm{O} Q$-value spectra measured under the same experimental conditions at $\theta_{\text {lab }}=5^{\circ}$ and incoming pion encrgy $T_{\pi}=295 \mathrm{MeV}$. The spectra have been corrected for the spectrometer acceptance as a function of momentum. The $\left(\pi^{-}, \pi^{+}\right)$data are from the present measurement and the $\left(\pi^{+}, \pi^{-}\right)$fit results from reanalysis of recent data from Ref. [2]. In addition to the weak transitions to the ground states of ${ }^{13} \mathrm{Be}$ and ${ }^{13} \mathrm{O}$, respectively, both spectra contain a wide peak labeled (GDR) $)^{2}$ located high in the continuum region. In the $\left(\pi^{+}, \pi^{-}\right)$rcaction the resonance is observed[2] at $Q=-46.5 \mathrm{MeV}$, but in the inverse reaction $\left(\pi^{-}, \pi^{+}\right.$) 


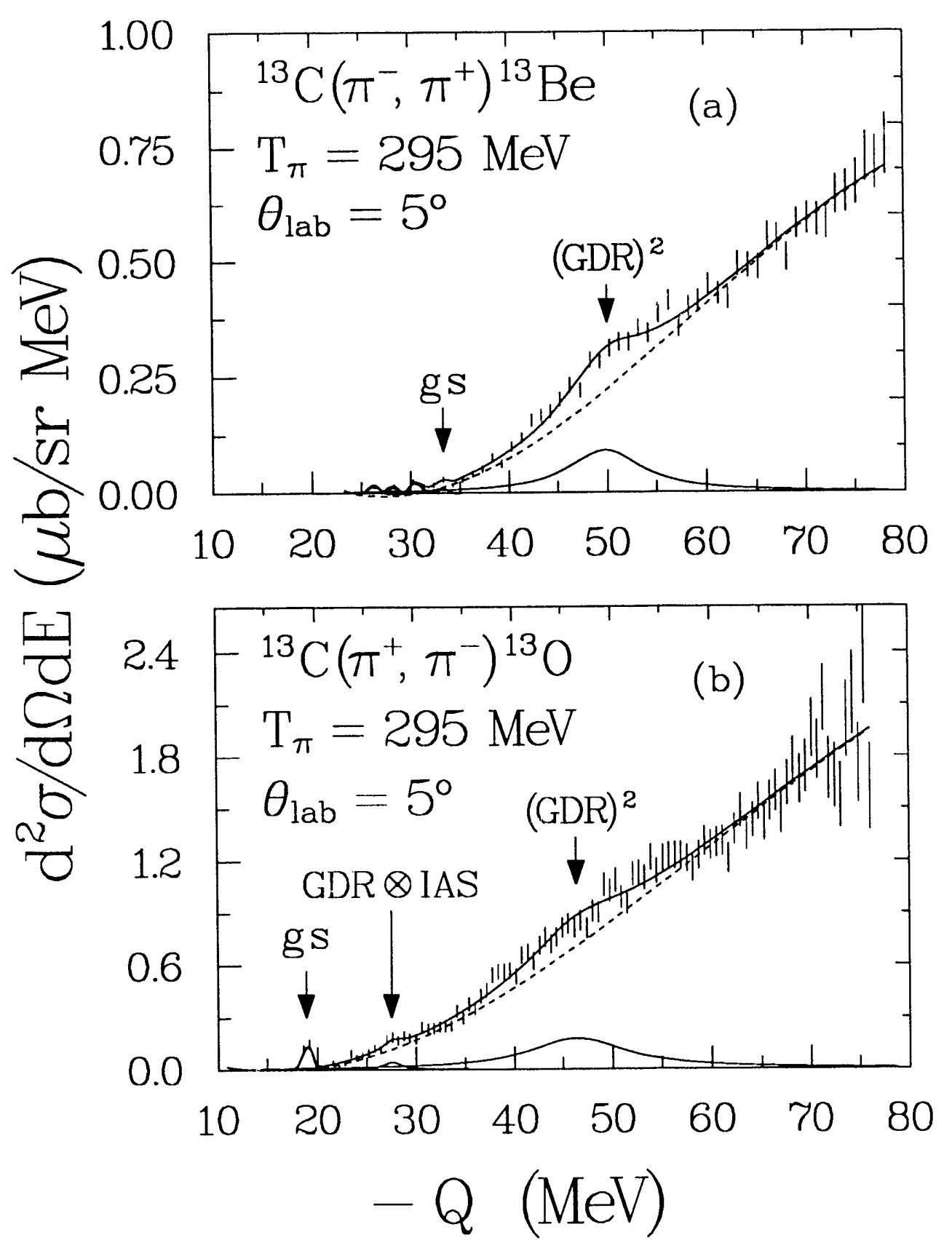

Figure 1: (a) Doubly differential cross-section spectrum for the ${ }^{13} \mathrm{C}\left(\pi^{-}, \pi^{+}\right)^{13}$ Be reaction at $T_{\pi}=295 \mathrm{MeV}$ and $\theta=5^{\circ}$. The arrows indicate the fitted location of the ground state (gs) and the giant resonance (GDR $)^{2}$. Short vertical lines represent statistical uncertainty of the data. The dashed line is the background fitted with a polynomial shape and the solid line is the fit to the spectrum using NEWFIT. (b) Same as (a) except for the ${ }^{13} \mathrm{C}\left(\pi^{+}, \pi^{-}\right)^{13} \mathrm{O}$ reaction. The arrows indicate the fitted location of the gs, the giant dipole built on the isobaric analog state (GDR $\otimes I A S)$, and the double giant dipole $\left(G^{2}{ }^{2}\right)$. 


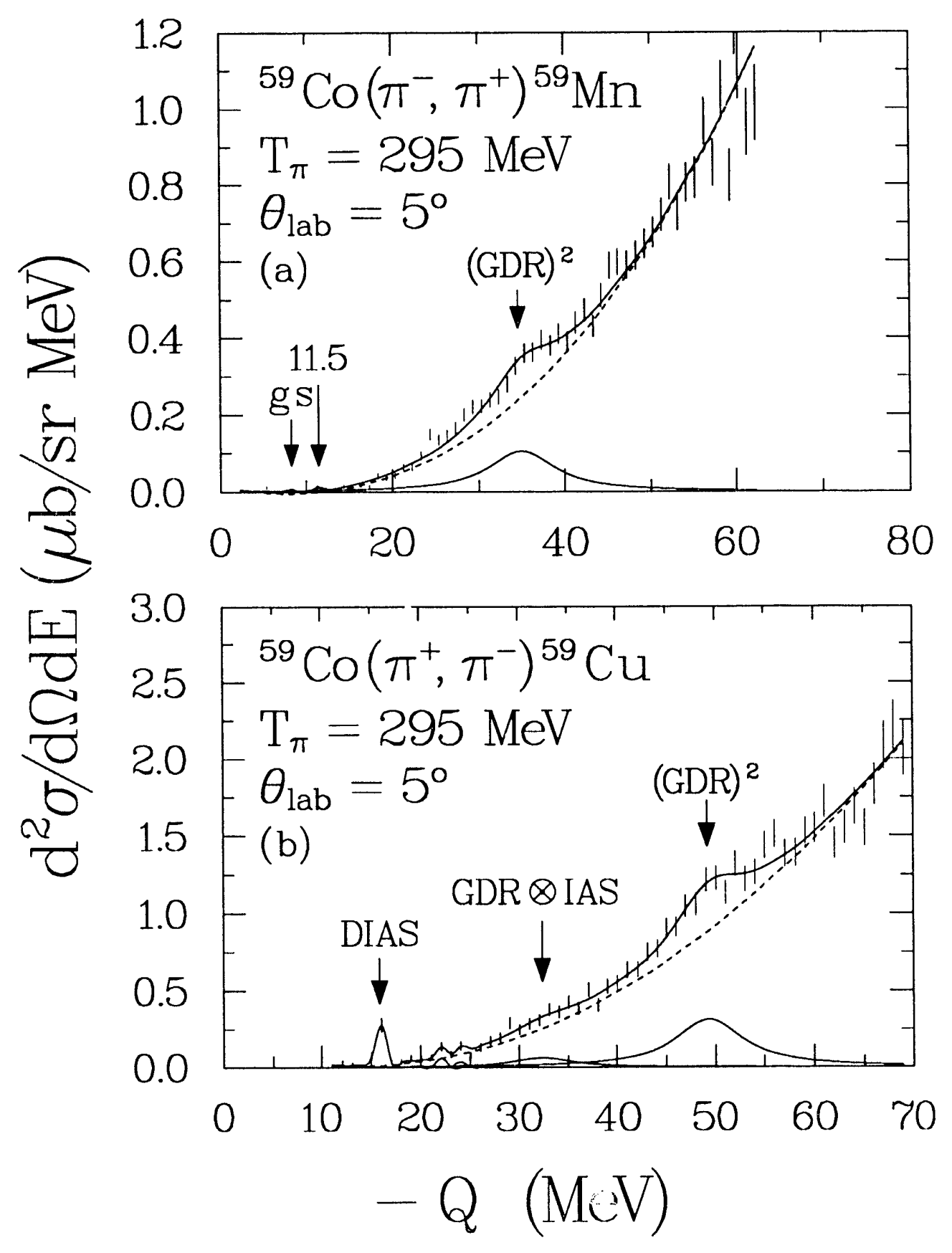

Figure 2: Same as Fig. 1 except for ${ }^{59}$ Co target. 
the resonance appears around $Q=-49.5 \mathrm{MeV}$. The energy relation between the two DCX modes is discussed in Section $B$. The giant resonance (GR) peaks were fitted with a Lorentzian shape of variable width. The fits in Fig. 1 use $\Gamma\left(G^{2} R^{2}\right)=9.0 \mathrm{MeV}$ for $\left(\pi^{-}, \pi^{+}\right)$and $12.0 \mathrm{MeV}$ for $\left(\pi^{+}, \pi^{-}\right)$. Figure $1(\mathrm{a})$ demonstrates also the existence of three low-lying states below the gs of ${ }^{13} \mathrm{Be}$. These weak transitions arise from the $10 \%{ }^{12} \mathrm{C}$ impurity in the ${ }^{13} \mathrm{C}$ target (Table $\mathrm{I}$ ) and correspond to the gs and two excited states at 2.1 and $4.6 \mathrm{MeV}$ in ${ }^{12} \mathrm{Be}$. $\mathrm{I}_{n}\left(\pi^{+}, \pi^{-}\right)$the corresponding states of ${ }^{12} \mathrm{O}$ are also present. They lie between the GDR $\otimes I A S$ and the $(G D R)^{2}$ in Fig. 1b. The backgrounds (clashed lines) which arise from DCX cross section to the continuum were fitted using a thircl-order. polynomial function of the $Q$ value: $\operatorname{Background}(Q, \theta)=a_{0}(\theta)+a_{1}(\theta) Q+a_{2}(\theta) Q^{2}+a_{3}(\theta) Q^{3}$, where $Q$ is the absolute $Q$ value. The same background form was used for both DCX modes. The solid lines are the resulting fits to the spectra. The resonance labeled (GDR) ${ }^{2}$ in the $\left(\pi^{+}, \pi^{-}\right)$reaction was previously[2] identified as the double isovector giant-clipole resonance. The strengths of the double dipoles reached in the $\left(\pi^{-}, \pi^{+}\right)$reactions and the ratios $\sigma_{\mathrm{DD}}\left(\pi^{-}, \pi^{+}\right) / \sigma_{\mathrm{DD}}\left(\pi^{+}, \pi^{-}\right)$will be discussed later. The lower GR observed in the $\left(\pi^{+}, \pi^{-}\right)$spectrum was identified[4] as the giant dipole built on the isobaric analog state and is labeled GDR $\otimes I A S$ in Fig. 1(b). This resonance is very weak at $5^{\circ}$ but appears very clearly at $\theta_{\text {lab }}=18^{\circ}$, an angle which corresponds to the peak of the dipole angular distribution at this energy[5]. The resonance is absent in the $\left(\pi^{-}, \pi^{+}\right)$spectrum, because no transition to the isobaric analog state (IAS) is allowed in this reaction mode. The computer program NEWFIT[6] was used to fit the spectra in these figures and to extract the parameters listed in Tables II and III.

Figure 2 displays the $\left(\pi^{-}, \pi^{+}\right)$and $\left(\pi^{+}, \pi^{-}\right) Q$-value spectra on ${ }^{59} \mathrm{Co}$. For $T \geq 2$ nuclei the double dipole in the $\left(\pi^{+}, \pi^{-}\right)$reaction splits into five isospin components. However in $\left(\pi^{-}, \pi^{+}\right)$, isospin considerations limit the reaction to only the upper isospin component $\left(T_{>}=T+2\right)$. These states are illustrated schematically in Fig. 3. Simple double-isospin coupling arguments give the strength ratios listed in the figure as $\left(\sum C_{1} C_{2}\right)^{2}$. Pauli-blocking effects will further suppress the upper isospin members of the double dipole in ${ }^{59} \mathrm{Cu}$. Therefore the observed resonance in the $\left(\pi^{+}, \pi^{-}\right)$reaction [Fig. 2(b)] contains mainly the three unresolved lower isospin components, whereas the double dipole in the $\left(\pi^{-}, \pi^{+}\right)$reaction [Fig. 2(a)] has only a single $(T=9 / 2)$ isospin component. An additional obvious difference between the spectra in Fig. 2 is the presence of the DIAS and the GDR $\otimes I A S$ transitions in the $\left(\pi^{+}, \pi^{-}\right)$spectrum. Both transitions are absent in the $\left(\pi^{-}, \pi^{+}\right)$mode. The spectra measured on the other targets are quite similar to those presented in Figs. 1 and 2 and are not shown here. The measured $Q$ values for the double dipole are given in Table II. Table III gives all the measured quantities for the double GDR ( $Q$ values, cross sections at $5^{\circ}$ and the widths) for both reaction modes. Also listed in Table III are the ratios of the double-dipole cross sections in $\left(\pi^{-}, \pi^{+}\right)$and $\left(\pi^{+}, \pi^{-}\right)$, the Coulomb displacencnt cnergies and the energy differences $\Delta E_{S}^{\prime}$ discussed later.

\subsubsection{Energy relations and symmetry energy}


$\mathrm{GDR}_{+}^{2}$

GDR_
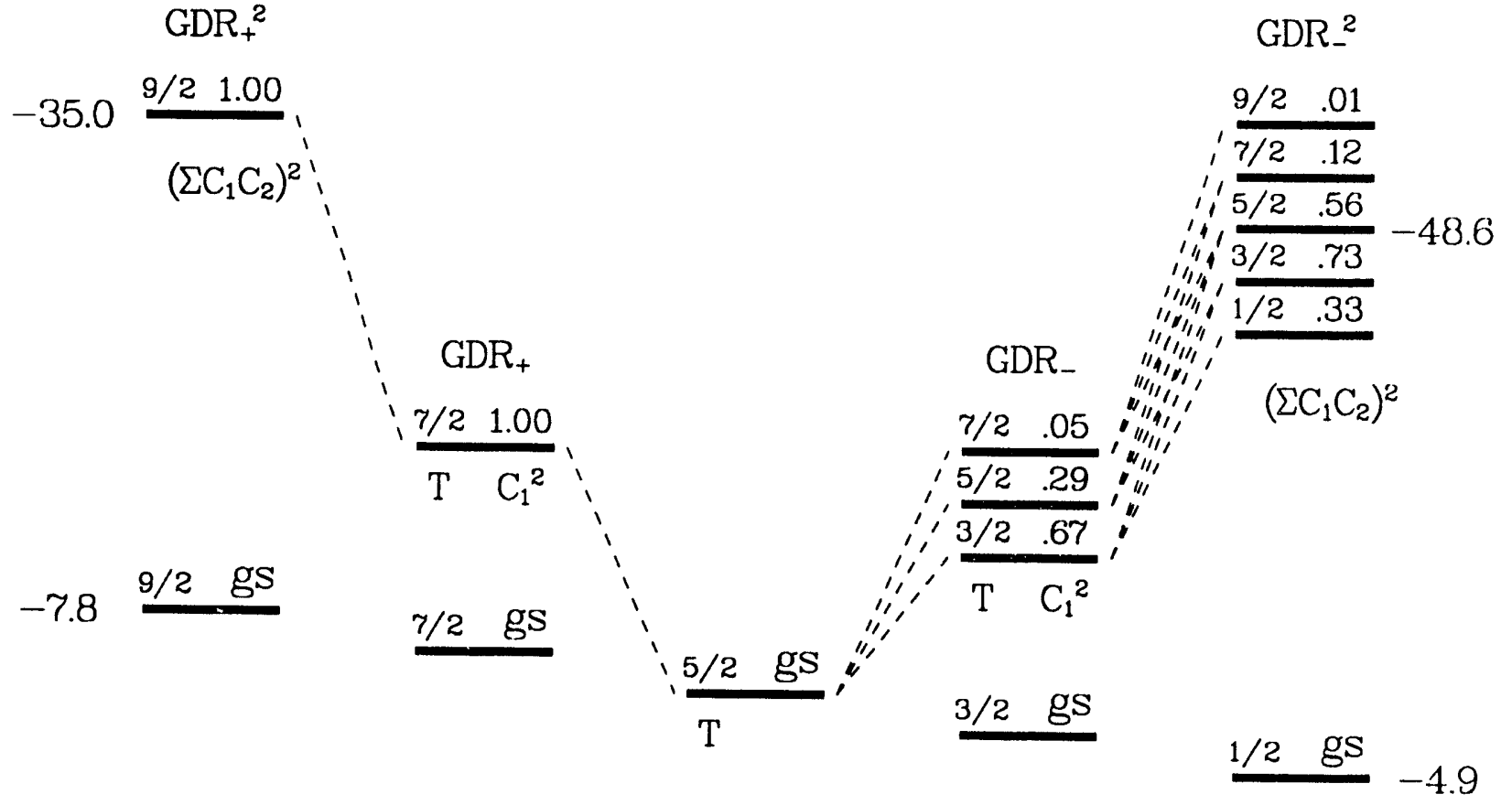

$\mathrm{Q}(\mathrm{MeV}){ }^{59} \mathrm{Mn}$

${ }^{59} \mathrm{Fe}$

${ }^{59} \mathrm{Ni}$

${ }^{59} \mathrm{Cu} \quad \mathrm{Q}(\mathrm{MeV})$

$\mathrm{T}_{\mathrm{Z}}=9 / 2$

$\mathrm{T}_{\mathrm{z}}=7 / 2$

$\mathrm{T}_{\mathrm{z}}=5 / 2$

$\mathrm{T}_{\mathrm{z}}=3 / 2$

$\mathrm{T}_{\mathrm{z}}=1 / 2$

Figure 3: Level scheme for DCX to the double isovector giant dipole resonances on ${ }^{59} \mathrm{Co}$. Vertical placement of states is not to scale. $C_{1}$ and $C_{2}$ are isospin Clebsch-Gordon coefficients, and the sums indicate double coupling coefficients. The listed $Q$ values on the right and left sides are from previous[2] and present experiments. 


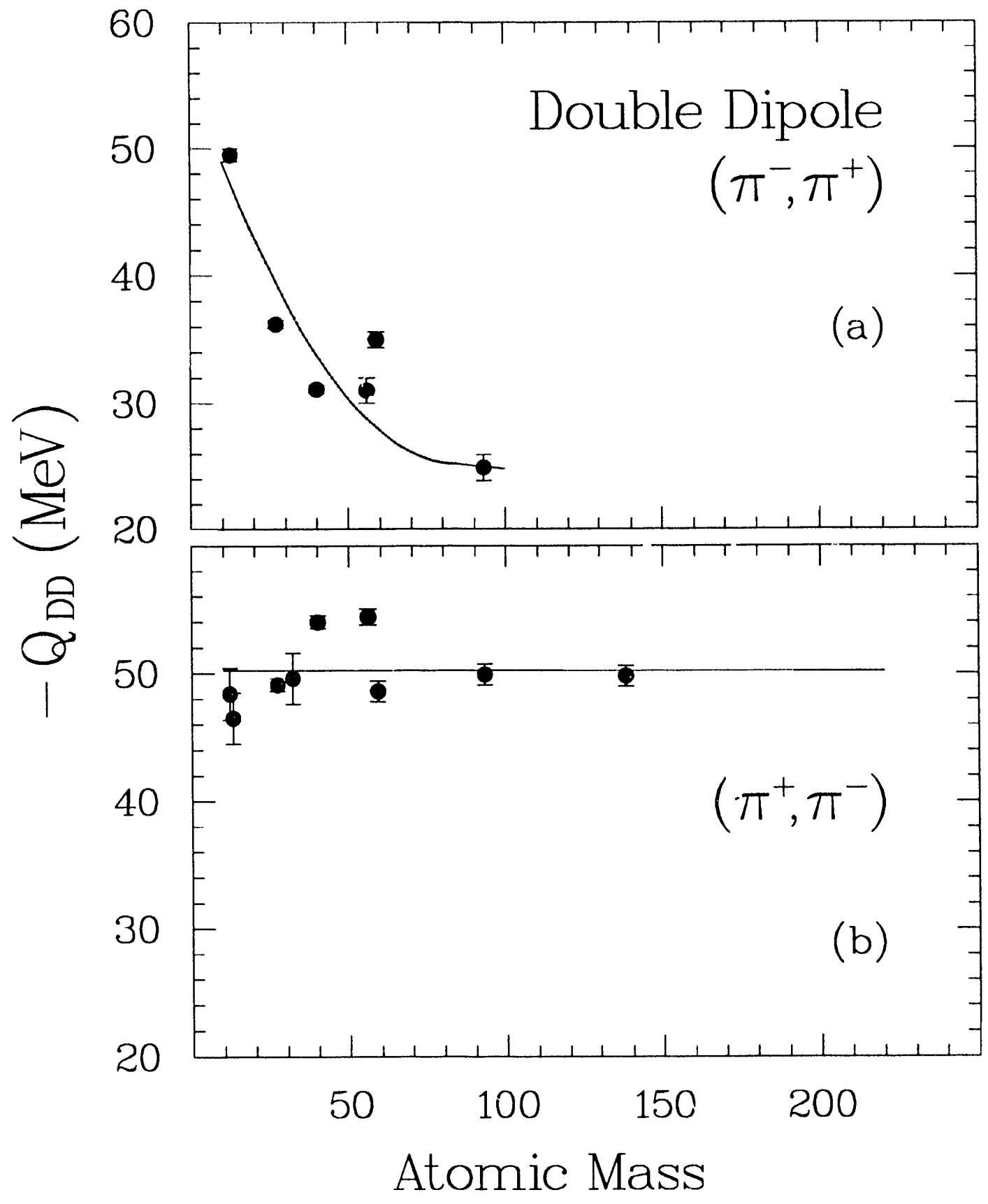

Figure 4: $Q$-values versus $A$ for the double dipole in both pion DCX modes. (a) $\left(\pi^{-}, \pi^{+}\right)$ data from the present study. The curve is to guide the eye. (b) $\left(\pi^{+}, \pi^{-}\right)$data from Rof. [2]. The solid line is the least-mean-square value. 
Figure 4 displays the observed $Q$ value for the double dipole as a function of atomic mass. In $\left(\pi^{+}, \pi^{-}\right)$the double GDR appears around $Q=-50 \mathrm{MeV}$ and its energy is almost independent of $A$. The doubly excited GDR in the $\left(\pi^{+}, \pi^{-}\right)$reaction is reached by adding two E1 vibrational energies and two Coulomb energies to the target nucleus gromd state. The E1 energy decreases with $A$, but the Coulomb energy increases with $Z$ and the two effects almost cancel each other, yielding a nearly constant $Q$ value for the double GDR excitations. Experimentally the single-charge-exchange GDR is observed in $\left(\pi^{+}, \pi^{0}\right)$ around $Q=-25 \mathrm{MeV}$ for a wide range of mass. Thus the energies of the double dipoles observed in $\left(\pi^{+}, \pi^{-}\right)$are close to double the energy of the "single" GDR, i.e.,

$$
Q_{\left(\pi^{+}, \pi^{-}\right)}\left(\mathrm{GDR}^{2}\right) \simeq 2 Q_{\left(\pi^{+}, \pi^{0}\right)}(\mathrm{GDR}) \simeq-50 \mathrm{MeV} .
$$

This near er uality is surprising because it neglects the effect of isospin splitting, which exists in both the $\left(\pi^{+}, \pi^{0}\right)$ and the $\left(\pi^{+}, \pi^{-}\right)$reactions, but is somewhat different in the two. The double GDR observed in $\left(\pi^{-}, \pi^{+}\right)$[Fig. 4(a)] has a significantly different trend. This state is obtained by adding two $\mathrm{E} 1$ vibrational energies to the nucleus and subtracting two Coulomb energies, since two protons are changed into two neutrons. Thus the $Q$ values in $\left(\pi^{-}, \pi^{+}\right)$are expected to decrease strongly (in absolute value) with $A$. The lowering of the magnicude of the double-dipole $Q$ value is very significant for medium and heavy nuclei. For example, the double dipole on ${ }^{93} \mathrm{Nb}$ has $Q=-49.9 \mathrm{MeV}$ in ${ }^{93} \mathrm{Nb}\left(\pi^{+}, \pi^{-}\right)^{93} \mathrm{Tc}$ but appears as low as $Q=-24.9 \mathrm{MeV}$ in the ${ }^{93} \mathrm{Nb}\left(\pi^{-}, \pi^{+}\right)^{93} \mathrm{Y}$ reaction. The solid line drawn in Fig. 4(a) is merely to guide the eye while the line in Fig. 4(b) is a least-square constant fit to the data which gives $Q=-50.2 \mathrm{MeV}$, for $12 \leq A \leq 197$.

The energy relation between the double dipole in the two DCX modes can be written as:

$$
Q_{\mathrm{DD}}\left(\pi^{-}, \pi^{+}\right)-Q_{\mathrm{DD}}\left(\pi^{+}, \pi^{-}\right)=4\left(\Delta E_{C}-\Delta m_{n p}\right)-\Delta E_{S}^{\prime},
$$

where $\Delta E_{C}$ is the average Coulomb displacement energy for the $T_{0}+2$ multiplet with $T_{z}=\left(T_{0}+2\right),\left(T_{0}+1\right), T_{0},\left(T_{0}-1\right)$ and $\left(T_{0}-2\right)$. The quantity $\Delta m_{n p}$ is the neutron-proton mass difference $(1.29 \mathrm{MeV})$. Eq. 2 then defines $\Delta E_{S}^{\prime}$ which is related to the symmetry energy of the single GDR[ 7$]$. We have used experimental values for the $T_{0}+1$ multipole Coulomb displacement energies when the neighboring states in the multiplet are known (up to mass 40). For heavier nuclei, only one pair of states is known. In order to avoid odd-even mass difference ambiguities we have used the relation[8]

$$
\Delta E_{C}=\frac{1.412 Z}{A^{1 / 3}}-0.861 \mathrm{MeV}
$$

where $Z$ and $A$ refer to the atomic and mass numbers, respectively, of the target nucleus. The Coulomb energies are listed in Table III.

Using Eq. 2 and the experimental $Q$ values listed in Table III, we obtain the $\Delta E_{S}^{\prime}$ values given in the table. These quantities give the energy differences between $T_{>}$and the centroid of the double dipole members reached in the $\left(\pi^{+}, \pi^{-}\right)$reaction. For ${ }^{13} \mathrm{C}$ the $T_{<}$and $T_{>}$components of the GDR have been measured in photonuclear studies [9]. The centroids of the two components are separated by $6.8 \mathrm{MeV}$ from which a Lane potential 
$[V=6.8 A /(T+1)]$ of $59 \mathrm{MeV}$ can be derived. This can then be used to calculate the expected lineshape for the double dipole in $\left(\pi^{+}, \pi^{-}\right)$, following the procedures outlined in Ref. [7]. The $10 \%$ contribution from the upper isospin component causes the extracted value of $\Delta E_{S}^{\prime}$ to shift to $8.7 \pm 2.0 \mathrm{MeV}$. The value of $\Delta E_{S}^{\prime}$ can be calculated knowing the Lane potential[7] giving an expected value of $10.3 \mathrm{MeV}$, which compares favorably with our measured value. Using the same Lane potential $(59 \mathrm{MeV})$ we expect $\Delta E_{S}^{\prime}$ for ${ }^{27} \mathrm{Al}$ to be $4.96 \mathrm{MeV}$, which compares with our extracted value of $2.7 \pm 0.6 \mathrm{MeV}$. A discrepancy is also apparent in the $T=0$ data. Here we expect $\Delta E_{S}^{\prime}=0$, but for ${ }^{40} \mathrm{C}$ a we extract $\Delta E_{S}^{\prime}=1.13 \pm 0.6 \mathrm{MeV}$, although here the deviation is in the other dircction. These deviations are not currently understood, but may be related to a poor estimate of the Coulomb displacement energy, or the assumption that the Lane potential is the same for single and double resonances. For heavier nuclei the values of $\Delta E_{S}^{\prime}$ for the doubly excited GDR deduced from Eq. 2 are expected to be much larger than for the single GDR since the states involved differ by several ( $\approx$ three or more) isospin units as illustrated in Fig. 3 , for the case of ${ }^{59} \mathrm{Co}$.

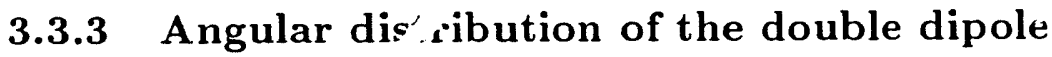

Figure 5 displays the angular distribution measured for the double dipole for both ${ }^{40} \mathrm{Ca}\left(\pi^{+}, \pi^{-}\right)^{40} \mathrm{Ti}$ (from Ref. [2]) and ${ }^{40} \mathrm{Ca}\left(\pi^{-}, \pi^{+}\right)^{40} \mathrm{Ar}$. The figure contains data from the present work for the ${ }^{40} \mathrm{Ca}\left(\pi^{-}, \pi^{+}\right)^{40} \mathrm{Ti}$ measurements at three laboratory scattering angles $5^{\circ}, 12^{\circ}$, and $19^{\circ}$. Figure 5 shows that the new partial angular distribution measured in $\left(\pi^{-}, \pi^{+}\right)$is nearly identical to the angular distribution measured earlier under the same experimental conditions in the $\left(\pi^{+}, \pi^{-}\right)$reaction. The dot-dash lines are the results of simple seciuentialmodel calculations using the pion coupled-channel impulse-approximation (CCIA) code NEWCHOP[10]. The calculations include the ground state (gs), the giant-dipole resonance (GDR), and the double dipole $\left(G^{2} R^{2}\right)$. The dashed lines are the calculations for the double dipole with $J^{\pi}=2^{+}$and the dotted lines are for $J^{\pi}=0^{+}$. The solid lines are the sums of the $0^{+}$and $2^{+}$curves with the normalization factors listed in the figure. The normalization factors for the $0^{+}$curves were chosen to account for the forward-angle rises of the cross sections when added to the $2^{+}$curves. Both angular distributions show a forward-angle rise which may indicate a contribution of the $J^{\pi}=0^{+}$double-dipole state[11]. The small (but non-zero) normalization factors for the $0^{+}$curves indicate that the observed resonances are primarily the $J^{\pi}=2^{+}$double-dipole states, but may indicate that we actually observe part of the unresolved $J^{\pi}=0^{+}$double-dipole strength.

\subsubsection{Width of the double-dipole resonance}

Figure 6 presents the widths of the double-dipole states observed in the $\left(\pi^{-}, \pi^{+}\right)$and $\left(\pi^{+}, \pi^{-}\right)$reactions. In $\left(\pi^{+}, \pi^{-}\right)$the width of the double dipole is around $10 \mathrm{MeV}$ and is larger than the width of the "single" dipole in $\left(\pi^{+}, \pi^{0}\right)\left[\left\langle\Gamma_{\mathrm{GDR}}\right\rangle=6.3 \pm 1.0 \mathrm{MeV}\right] \mathrm{by}$ a factor of about 1.5[12]. This disagrees somewhat with theoretical estimates that the width of the double GDR, as a two-phonon state, should be twice the GDR width[2,13]. The solid 


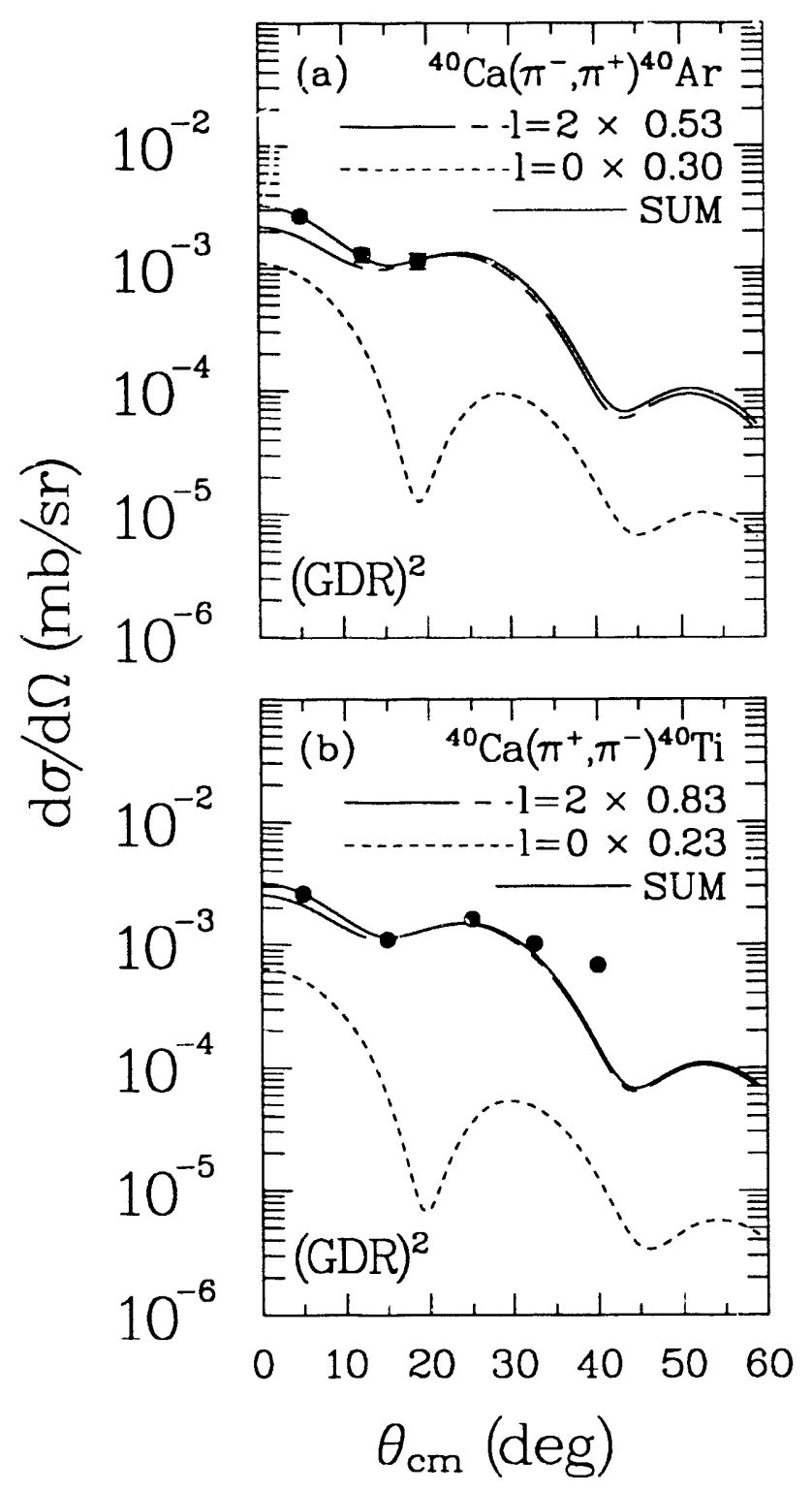

Figure 5: Angular distribution for the resonances identified as the double dipoles in the ${ }^{40} \mathrm{Ca}\left(\pi^{ \pm}, \pi^{ \pm}\right)$reactions at $T_{\pi}=295 \mathrm{MeV}$. The curves are sequential model calculations for the double dipole using NEWCHOP[10]. The dashed line is an $l=2$ quadrupole distribution normalized to fit the $19^{\circ}$ (a) and $15^{\circ}$ (b) data points. The dotted lines are $l=0$ curves normalized such that the sum of the $l=2$ and $l=0$ distributions fits the forward angle cross sections. 


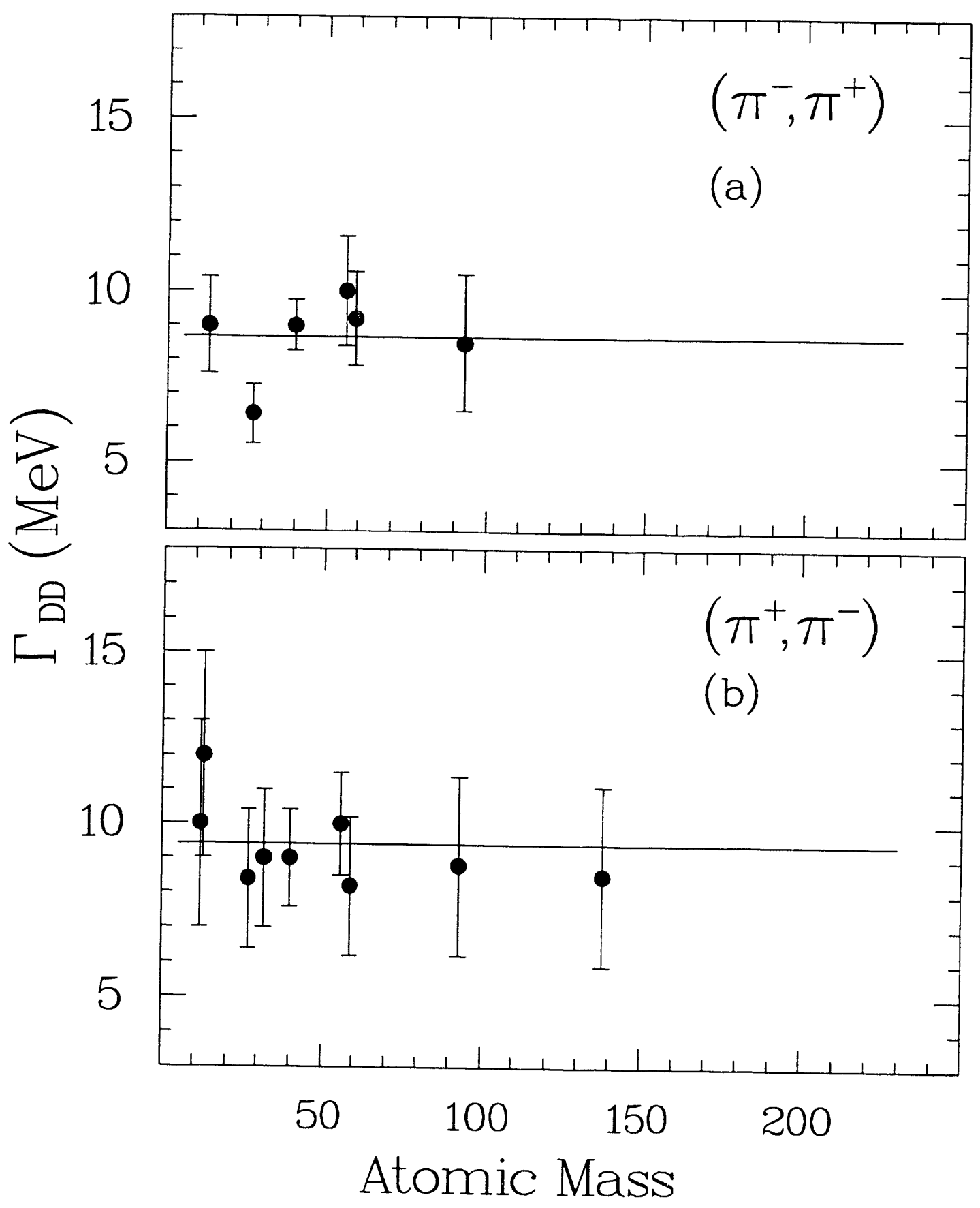

Figure 6: Widths of the double isovector giant dipole state as a function of $A$ for both DCX modes. The solid lines in (a) and (b) are least-mean-square values. The widths were obtained using NEWFIT and a Lorentzian line shape for the resonances as illustrated in Figs. 1 and 2. 
lines are the weighted averages of the data, if the widths in a given mode are independent of $A$. For $\left(\pi^{+}, \pi^{-}\right)$the weighted average is $\left\langle\Gamma_{\mathrm{DD}}\right\rangle=9.23 \pm 0.76 \mathrm{MeV}$ and for $\left(\pi^{-}, \pi^{+}\right)$ $\left\langle\Gamma_{\mathrm{DD}}\right\rangle=8.60 \pm 0.51 \mathrm{MeV}$. In $\left(\pi^{-}, \pi^{+}\right)$our average widths are about twice the average widths measured for the single dipole in $\left(\pi^{-}, \pi^{0}\right)\left\langle\Gamma_{\mathrm{GDR}}\right\rangle=4.2 \pm 1.0 \mathrm{MeV}$ [12]. The double dipole on ${ }^{27} \mathrm{Al}$ in $\left(\pi^{-}, \pi^{+}\right)$has a width of only $6.4 \pm 1.0 \mathrm{MeV}$ and thus is overestimated by the above constant average. In the $\left(\pi^{-}, \pi^{+}\right)$reaction the double dipole has only a single isospin component, and therefore is expected to be narrower than in $\left(\pi^{+}, \pi^{-}\right)$where the observed resonance is composed of two to five isospin components. Table III summarizes the deduced widths of the double-dipole resonances observed in the two DCX modes.

\subsubsection{Cross-section ratios and background analysis}

Figure 7 presents the cross-section ratios for the double-dipole resonances reached in $\left(\pi^{-}, \pi^{+}\right)$and $\left(\pi^{+}, \pi^{-}\right)$reactions on the same target nuclei. For all nuclei with $N>Z$ studied in the present work $\left({ }^{13} \mathrm{C},{ }^{27} \mathrm{Al},{ }^{56} \mathrm{Fe},{ }^{59} \mathrm{Co}\right.$, and $\left.{ }^{93} \mathrm{Nb}\right)$ the cross section in $\left(\pi^{-}, \pi^{+}\right)$ is weaker than in $\left(\pi^{+}, \pi^{-}\right)$. The suppression of the $\left(\pi^{-}, \pi^{+}\right)$cross sections is nearly independent of atomic mass, but the ratio may decrease slightly with $A$. The effect of Pauli blocking in changing two protons into two neutrons is expected to increase in neutron-rich nuclei. A similar effect was found for the $1 \hbar \omega$ excitation of the GDR in pion single-chargeexchange[12]. The deduced cross-section ratios $R\left(\mathrm{GDR}^{2}\right)=\sigma_{\mathrm{DD}}\left(\pi^{-}, \pi^{+}\right) / \sigma_{\mathrm{DD}}\left(\pi^{+}, \pi^{-}\right)$are listed in Table III. We will return to the ${ }^{40} \mathrm{Ca}$ case later.

Table IV compares the cross-section ratios from DCX with the cross-section ratios $R(\mathrm{GDR})=\sigma_{\mathrm{GDR}}\left(\pi^{-}, \pi^{0}\right) / \sigma_{\mathrm{GDR}}\left(\pi^{+}, \pi^{0}\right)$ for the "single"-dipole excitation from pion SCX data[12]. Since no data are available for SCX on ${ }^{59} \mathrm{Co}$ and ${ }^{93} \mathrm{Nb}$ we used in Table IV data for the nearest nuclei $\left({ }^{60} \mathrm{Ni}\right.$ and ${ }^{90} \mathrm{Zr}$ ) for which data are available. Also listed in the table are the theoretical predictions for the cross-section ratios for exciting the GDR in $\left(\pi^{-}, \pi^{0}\right)$ and $\left(\pi^{+}, \pi^{0}\right)$ using transition densities from Hartree-Fock-random-phase-approximation calculations[14]. No theoretical work has been reported for the Pauli-blocking effect in $\left(\pi^{-}, \pi^{+}\right)$compared with $\left(\pi^{+}, \pi^{-}\right)$transition strengths. However, the deduced cross-section ratios for the double dipole in DCX from the present study seem to be in good agrecment with the SCX data and the theoretical predictions. For example, for nuclei with large neutron excess, one would expect the blocking effect in DCX to be roughly the square of the corresponding attenuation factor in SCX, i.e.,

$$
R\left(\mathrm{GDR}^{2}\right)=[R(\mathrm{GDR})]^{2}
$$

since in the simplest picture, pion DCX can be viewed as two sequential SCX processes. This estimate seems to be close to the measured cross-section ratio for the double dipole on ${ }^{93} \mathrm{Nb}: R\left(\mathrm{GDR}^{2}\right)=0.23 \pm 0.09$ in the present study. For lighter nuclei one would expect $R\left(\mathrm{GDR}^{2}\right)$ to be somewhere between $R(\mathrm{GDR})$ and $[R(\mathrm{GDR})]^{2}$ as observed for ${ }^{59} \mathrm{Co}$. In the very light nuclei ${ }^{13} \mathrm{C}$ and ${ }^{27} \mathrm{Al}$ the measured cross-section ratio of $\left(\pi^{-}, \pi^{+}\right)$to $\left(\pi^{+}, \pi^{-}\right)$is however unexpectedly small.

The nucleus ${ }^{40} \mathrm{Ca}$ is an exceptional case in which the double-dipole cross section in $\left(\pi^{-}, \pi^{+}\right)$is larger than in $\left(\pi^{+}, \pi^{-}\right)$. This result might be due to the differences in the 


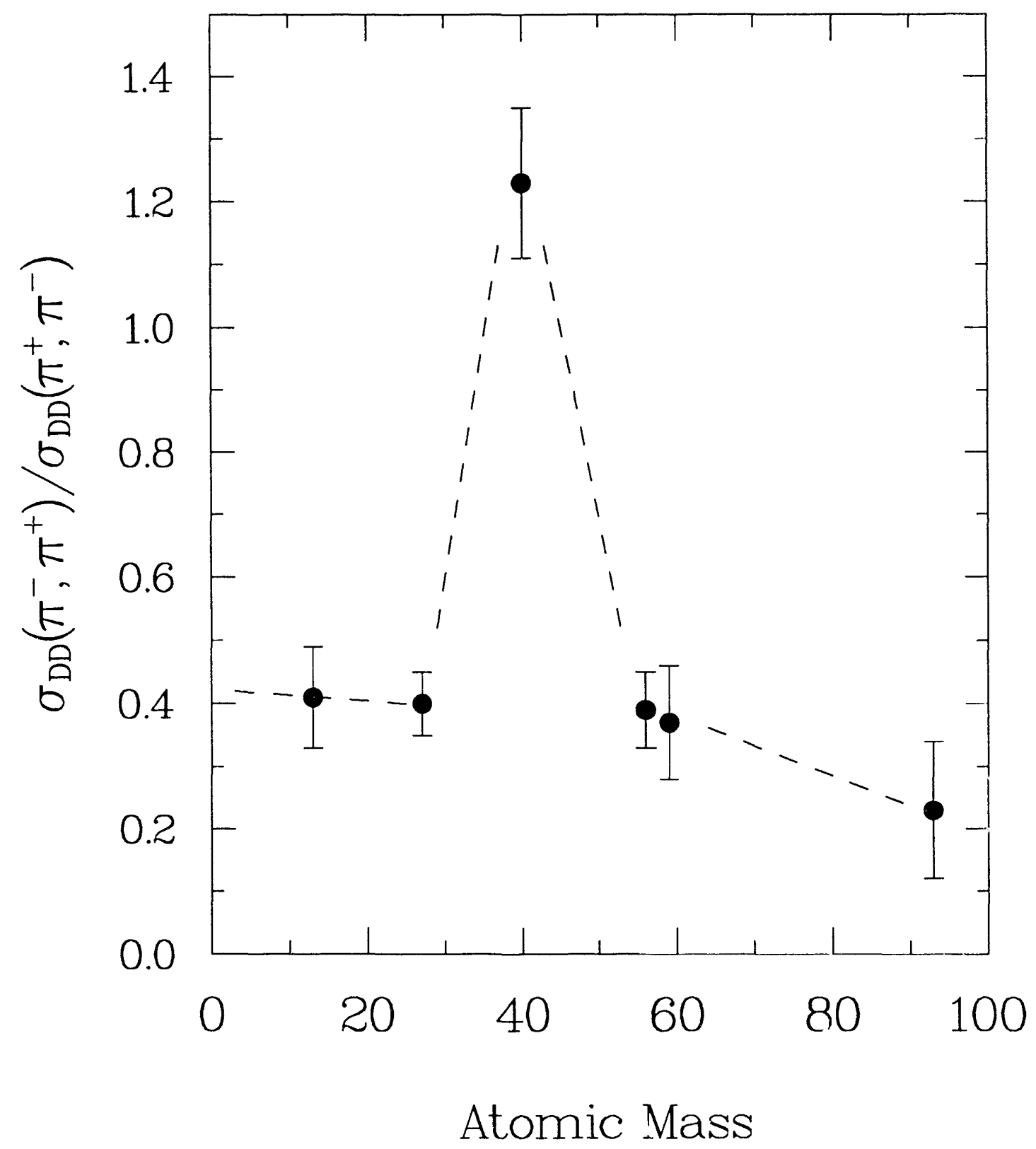

Figure 7: Ratio of the double dipole cross sections in $\left(\pi^{-}, \pi^{+}\right)$to $\left(\pi^{+}, \pi^{-}\right)$taken from Talble III plotted as a function of $A$. The dashed line is to guide the eye. 
spatial distributions of the protons and neutrons in the self-conjugate ${ }^{40} \mathrm{Ca}$ nucleus. It is well known that, because of the Coulomb interaction, the protons in ${ }^{40} \mathrm{Ca}$ are expelled relative to the neutrons. In an $N=Z$ nucleus such as ${ }^{40} \mathrm{Ca}$ the Coulomb interaction causes a slight excess of the proton density at the surface of the nucleus. Hartree-Fock calculations, $f_{\mathrm{OI}}$ example, give a root-mean-square radius for the proton distribution $\left\langle r^{2}\right\rangle_{p}^{1 / 2}$ larger by about $1 \%$ than the rms radius of the neutron distribution[15]. This slight excess of protons at the surface of ${ }^{40} \mathrm{Ca}$ could cause the $\left(\pi^{-}, \pi^{+}\right)$cross sections to be larger than the $\left(\pi^{+}, \pi^{-}\right)$ cross sections. A similar increase of the GDR cross section was also observed in $\left(\pi^{-}, \pi^{0}\right)$ compared with $\left(\pi^{+}, \pi^{0}\right)$ in SCX on ${ }^{40} \mathrm{Ca}$ (Table IV); however in pion DCX the effect scems to be more dramatic - as discussed next in the background analysis.

Figure 8 combines all background spectra from the two DCX modes. The background arises from DCX cross section to the continuum of states encountered in the nucleus at high excitation energies. Some remarkable features are observable in these spectra. For a given $A$ the cross section to the continuum increases with $Q$ value-due (most likely) to the increase in the density of states with increasing excitation energy in the final nucleus. Furthermore, for a given $Q$ value, the background level increases with $A$, because of the increase in the number of neutrons and protons available for DCX reactions. However, there is a clear irregularity in both Fig. 8(a) and Fig. 8(b). In the $\left(\pi^{-}, \pi^{+}\right)$reaction [Fig. 8(a)] the ${ }^{40} \mathrm{Ca}$ background cross section is the highest, and has the largest overall DCX cross section of any nucleus up to ${ }^{93} \mathrm{Nb}$. On the other hand, in the $\left(\pi^{+}, \pi^{-}\right)$reaction [Fig. $8(\mathrm{~b})$ ] ${ }^{40} \mathrm{Ca}$ is again out of order, but now has the smallest background cross section. It seems very likely that this remarkable irregularity arises from the Coulomb polarization effects in the initial and final states as mentioned earlier. Around the $(3,3)$ resonance the pions probe mainly the surface of the nucleus, and therefore DCX is most sensitive to the nuclear tail, amplifying the influence of the Coulomb effects which cause an excess of protons on the nuclear surface of ${ }^{40} \mathrm{Ca}[15]$. This will enhance the $\left(\pi^{-}, \pi^{+}\right)$cross section and suppress the $\left(\pi^{+}, \pi^{-}\right)$cross section on ${ }^{40} \mathrm{Ca}$ as indeed observed experimentally. This feature is shown more dramatically in Fig. 9 where the background cross section is plotted versus $A$ for different $5-\mathrm{MeV}$ energy bins. Figure $9(\mathrm{a})$ presents the $\left(\pi^{-}, \pi^{+}\right)$, and Fig. $9(\mathrm{~b})$ the $\left(\pi^{+}, \pi^{-}\right)$ data. The irregularity of the ${ }^{40} \mathrm{Ca}$ case is now more obvious. The cross sections for the different energy bins increase with $A$ except for a local maximum for ${ }^{40} \mathrm{Ca}$ in $\left(\pi^{-}, \pi^{+}\right)$for all energy bins, and a local minimum in the inverse $\left(\pi^{+}, \pi^{-}\right)$reaction.

These features seem to be consistent with the available inclusive $\mathrm{DCX}$ data on ${ }^{40} \mathrm{Ca}$ and ${ }^{16} \mathrm{O}$ at $240 \mathrm{MeV}[16,17]$. The inclusive spectra on ${ }^{40} \mathrm{Ca}$ have a larger overall cross section than on ${ }^{16} \mathrm{O}$ in the $\left(\pi^{-}, \pi^{+}\right)$reaction, but have comparable cross sections in the $\left(\pi^{+}, \pi^{-}\right)$ reaction in the $Q$-value region covered by the present study. These features demonstrate again the overall enhancement of $\left(\pi^{-}, \pi^{+}\right)$over $\left(\pi^{+}, \pi^{-}\right)$cross sections on ${ }^{40} \mathrm{Ca}$. For ${ }^{16} \mathrm{O}$ this effect is found to be much smaller, as would be expected for a light nucleus. 


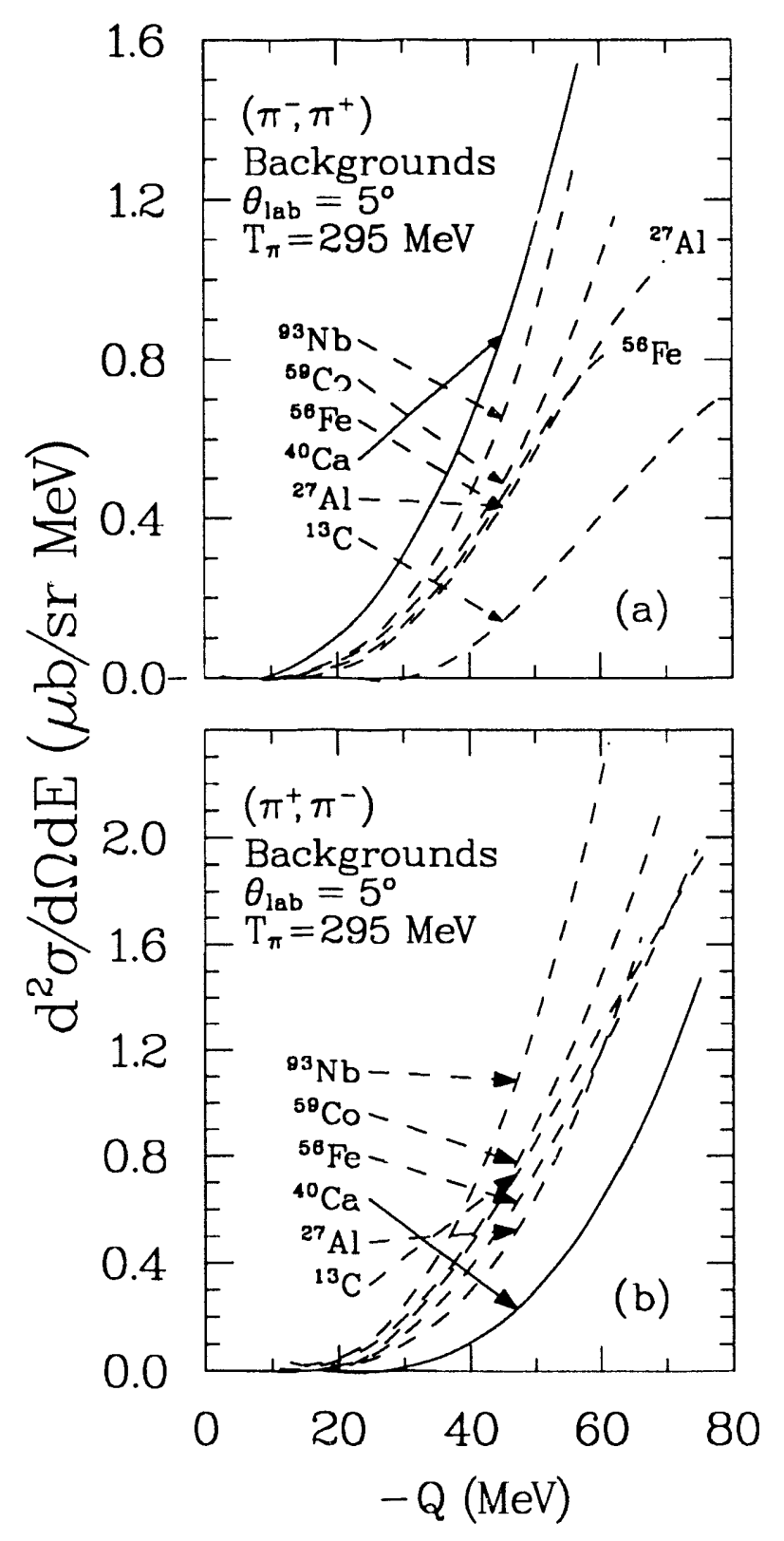

Figure 8: (a) Fitted background spectra for all targets studied in the $\left(\pi^{-}, \pi^{+}\right)$reaction in the present work at $5^{\circ}$ and $T_{\pi}=295 \mathrm{MeV}$. The background has been generated using i polynomial shape with use of NEWFIT as shown by the dashed lines in Figs. 1 and 2. (b) Same as (a) but for the inverse $\left(\pi^{+}, \pi^{-}\right)$reaction studied earlier Ref. [2]. 

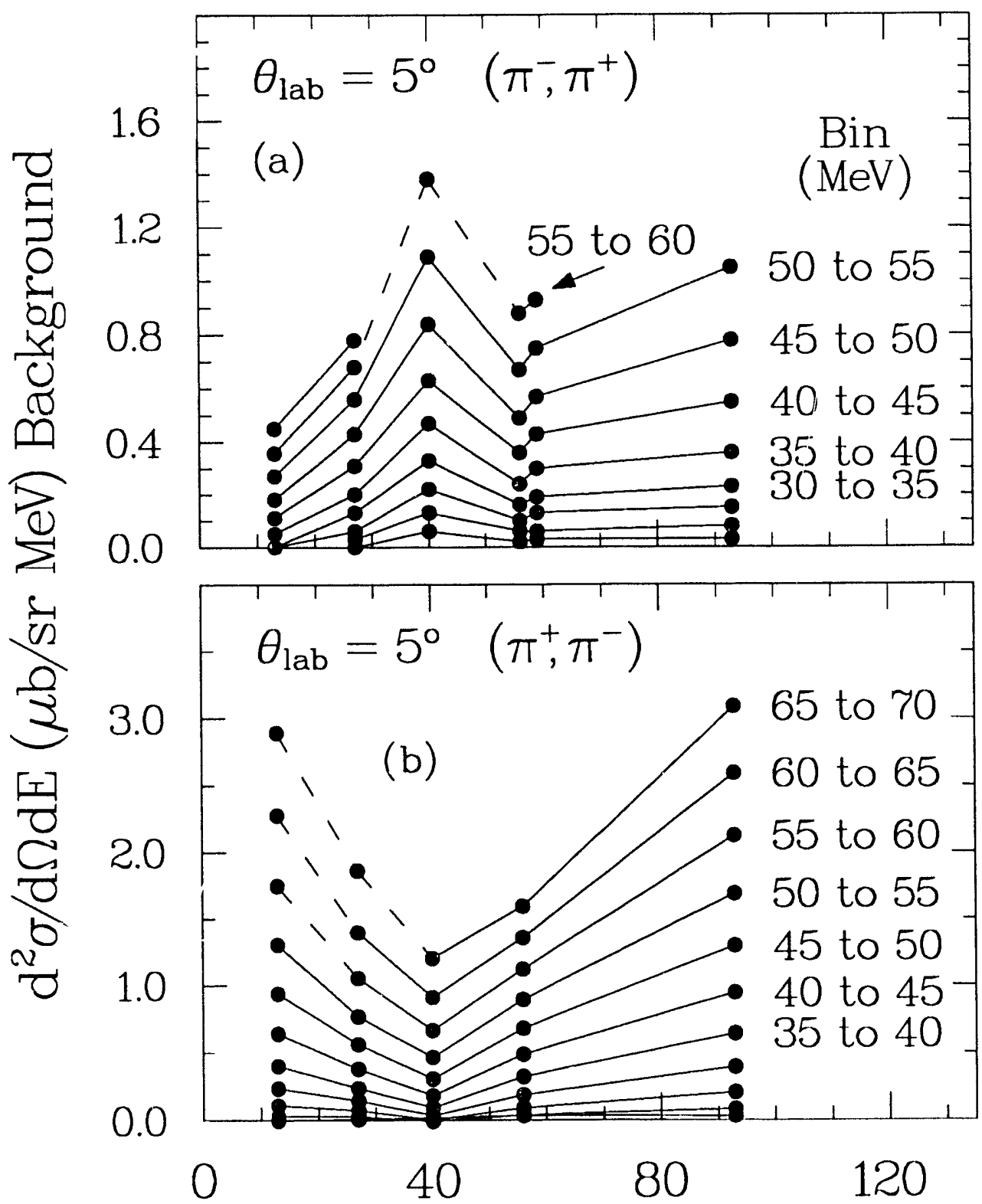

\section{Atomic Mass}

Figure 9: Doubly differential cross sections of the background spectra from Fig. 8 plotted versus $A$ for different $5-\mathrm{MeV} Q$-value bins. The data in (a) are from the present stucly, while those in (b) are from Ref. [2]. The dashed lines at some higher energy bins are extrapolations from the measured spectra. 


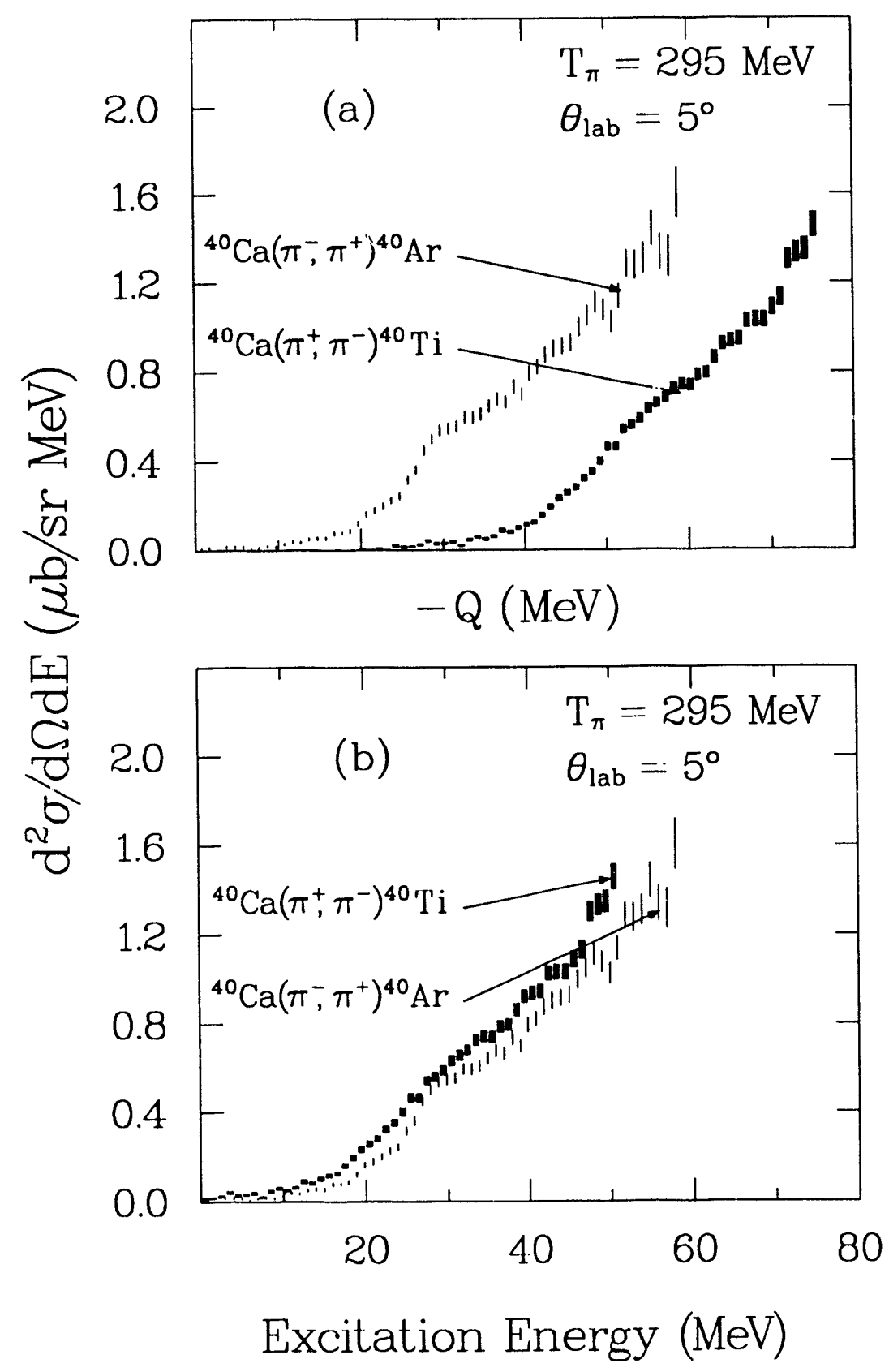

Figure 10: (a) Comparison between the doubly differential cross-section spectra for the ${ }^{40} \mathrm{Ca}\left(\pi^{-}, \pi^{+}\right)^{40} \mathrm{Ar}$ and the ${ }^{40} \mathrm{Ca}\left(\pi^{+}, \pi^{-}\right)^{40} \mathrm{Ti}$ reactions at $T_{\pi}=295 \mathrm{MeV}$ and $\theta_{\mathrm{lab}}=5^{\circ}$ plotted as a function of $Q$ value. The short vertical thin and thick lines represent the statistical uncertainty of the data, respectively. (b) Same as (a) except for plotting the two spectra as a function of excitation energy in the final mirror nuclei. 
In the above analysis we compared only spectra from the same DCX mode. One can also try to compare spectra from both $\left(\pi^{+}, \pi^{-}\right)$and $\left(\pi^{-}, \pi^{+}\right)$reactions on the same target nucleus. For example, Fig. 10 presents such a comparison for the self-conjugate ${ }^{40} \mathrm{C}^{\mathrm{C}} \mathrm{a}$ nucleus. Fig. 10(a) shows the data for $\left(\pi^{+}, \pi^{-}\right)$and $\left(\pi^{-}, \pi^{+}\right)$on ${ }^{40} \mathrm{Ca}$ as a function of $Q$ value. The $\left(\pi^{+}, \pi^{-}\right)$cross sections are substantially lower than the $\left(\pi^{-}, \pi^{+}\right)$cross scctions for given $Q$ values. To understand this phenomenon, we should remember that the same $Q$ value in the two reactions corresponds to very different excitation energies in the final ${ }^{40} \mathrm{Ti}$ and ${ }^{40} \mathrm{Ar}$ nuclei, and thus to different densities of states in these nuclei. (Note that, the total Coulomb displacement energy in ${ }^{40} \mathrm{Ti}-{ }^{40} \mathrm{Ar}$ is about $28 \mathrm{MeV}$.) If we correct for this effect by aligning the corresponding ground states (i.e., plotting the cross sections as a function of excitation energy), the two spectra come very close as expected from charge symmetry. This is shown in Fig. 10(b). However, as can be seen from Fig. 10(b), there are still small differences in the two spectra, which should be expected because after all the Coulomb interaction does break charge symmetry to some degree. For example, in the ${ }^{40} \mathrm{C}_{\mathrm{a}}$ target nucleus, as we already mentioned, the protons are slightly expelled with respect to the neutrons. Furthermore, there are kinematical charg" symmetry breaking effects such as the dependence of the $t$-matrices on the effective energy of the outgoing projectile which will produce differences in the two types of reactions. A detailed theoretical study of this effect is of considerable interest. We are able to make this kind of straightforward comparison in the case of ${ }^{40} \mathrm{Ca}$ in which we are dealing with mirror final nuclei, and there are no contributions from the symmetry energy. Not so are the $N-Z>0$ target nuclei, where the final nuclei are not charge conjugates and the comparison is more complicated because of the isospin splitting caused in the $\left(\pi^{+}, \pi^{-}\right)$reactions by the symmetry cnergy, and the differences in the available configuration space for the two DCX modes.

\subsubsection{Mass dependence of the double dipole}

Figure 11 compares the mass dependence of the cross sections for the double-dipole resonance in the two DCX modes. This issue was addressed in detail in Ref. [2] where we derive a semiempirical relation which gives the peak cross section for the double dipole in $\left(\pi^{+}, \pi^{-}\right)$. There, the double-dipole cross section is written as a produci of the strength of the first and second step GDR times an overall attenuation factor for DCX:

$$
\left(\frac{d \sigma}{d \Omega}\right)_{\mathrm{GLR}^{2}} \approx\left(\frac{N Z}{A^{2 / 3}}\right)^{2} A^{-\alpha^{\prime}} .
$$

The $\left(\pi^{-}, \pi^{+}\right)$cross sections seem to be consistent with those for $\left(\pi^{+}, \pi^{-}\right)$, but with a larger $A$ dependent attenuation factor. Using the above equation and the data of Fig. 11 we obtain $\alpha^{\prime}=2.35 \pm 0.05$ for $\left(\pi^{+}, \pi^{-}\right)$but $\alpha^{\prime}=2.54 \pm 0.10$ for $\left(\pi^{-}, \pi^{+}\right)$. The data in Fig. 11(a) for $\left(\pi^{-}, \pi^{+}\right)$are fit by $(\mathrm{d} \sigma / \mathrm{d} \Omega)_{\mathrm{DD}}\left(5^{\circ}\right)=(13400 \pm 1100)(N Z)^{2} A^{-3.87}(\mathrm{nb} / \mathrm{sr})$ and those in Fig. 11(b) for $\left(\pi^{+}, \pi^{-}\right)$are fit by $(\mathrm{d} \sigma / \mathrm{d} \Omega)_{\mathrm{DD}}\left(5^{\circ}\right)=(14130 \pm 500)(N Z)^{2} A^{-3.68}(\mathrm{nb} / \mathrm{si})$. The data for $\left(\pi^{+}, \pi^{-}\right)$is from Ref. [2], except for the recent ${ }^{27} \mathrm{Al}$ data point[3] which has becn added to the fit. The fit for $\left(\pi^{+}, \pi^{-}\right)$does not include the data points for ${ }^{12} \mathrm{C}$ and ${ }^{32} \mathrm{~S}$ 


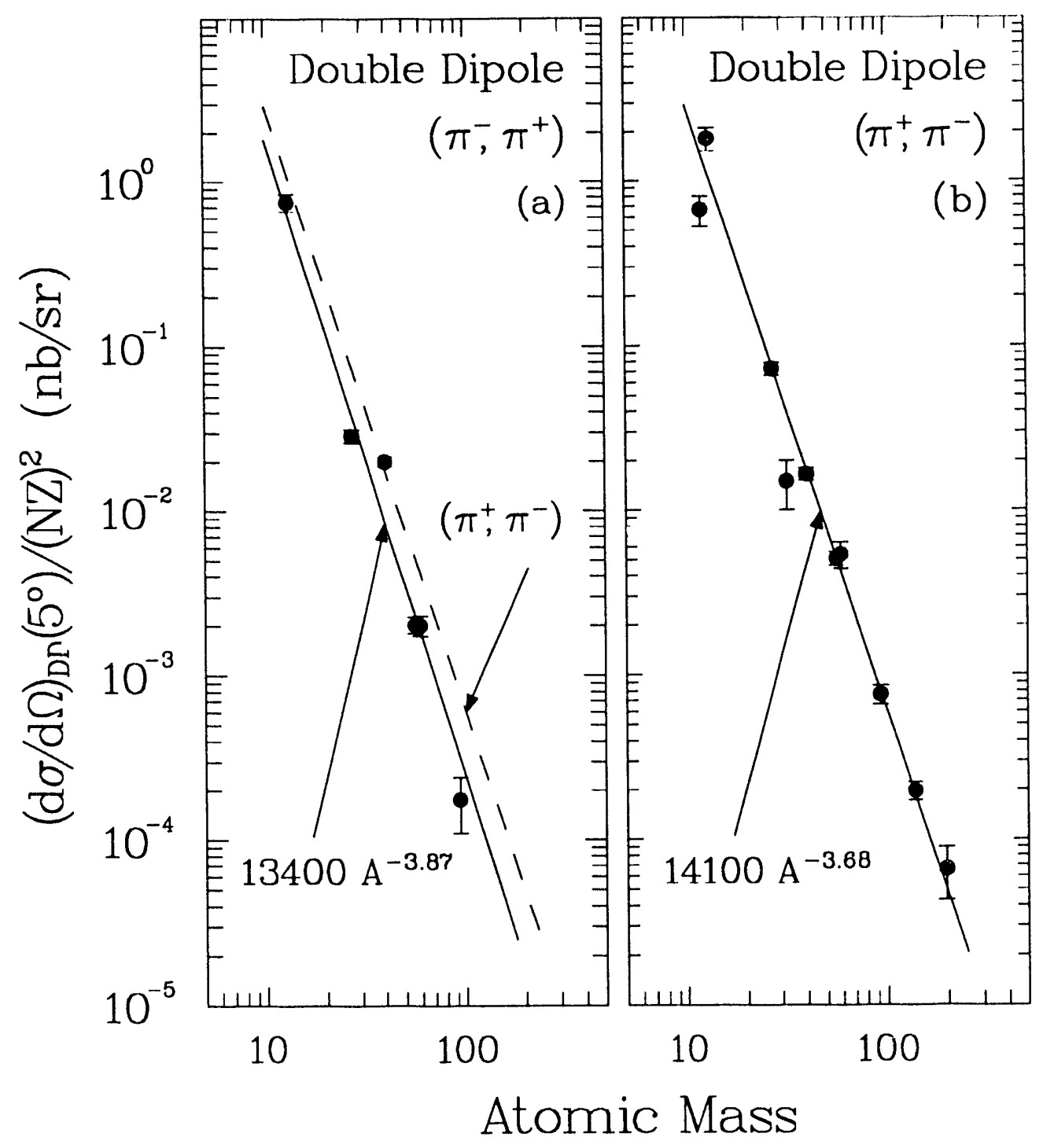

Figure 11: Plots of the double dipole cross sections at $5^{\circ}$ divided by $(N Z)^{2}$ vs $A$ for the two DCX modes. The data of (a) are from the present study, and the data of (b) are from Ref. [2]. Both (a) and (b) contain nearly superposed pairs of data points for ${ }^{56} \mathrm{Fe}$ and ${ }^{59} \mathrm{Co}$. The solid lines are power-law fits to the data with the parameters indicated in the figure. The dashed line in (a) is the same as the solid line in (b) for simple comparison of the two DCX modes. 
deformed nuclei. Fitting $\left(\pi^{+}, \pi^{-}\right)$results only for the same nuclei as in Fig 11(a) gives $(\mathrm{d} \sigma / \mathrm{d} \Omega)_{\mathrm{DD}}\left(5^{\circ}\right)=(19200 \pm 900)(N Z)^{2} A^{-3.77}(\mathrm{nb} / \mathrm{sr})$.

\section{References}

[1] S. Mordechai, et. al., Phys. Rev. Lett. 61, 531 (1988).

[2] S. Mordechai, et al., Phys. Rev. C 41, 202 (1990).

[3] S. Mordechai, et al., Phys. Rev. C 43, R1509 (1991).

[4] S. Mordechai et al., Phys. Rev. C 40, 850 (1989).

[5] S. Mordechai et al., Phys. Rev. C' 43, 1111 (1991).

[6] C. L. Morris (1986) unpublished.

[7] J. M. O'Donnell and H. T. Fortune, Phys. Rev. C (in press) 1991.

[8] M. S. Antony, J. Britz, and A. Pape, At. Data Nucl. Data Tables

[9] D. Zubanov, R. A. Sutton, M. N. Thompson, J. W. Jury, Phys. Rev. C 27, 1957 (1983).

[10] E. Rost, computer code CHOPIN (unpublished). The code has been modified by one of us (C.L.M.) to calculate pion charge-exchange reactions and renamed NEWCHOP.

[11] J. M. O'Donnell, H. T. Fortune and E. Rost, Phys. Rev. C' (in press) 1991.

[12] A. Erell, J. Alster, J. Lichtenstadt, M. A. Moinester, J. D. Bowman, M. D. Cooper', F. Irom, H. S. Matis, E. Piasetzky, and U. Sennhauser, Phys. Rev. C 34, 1822 (1986).

[13] N. Auerbach, Ann. Phys. 197, 376-395 (1990).

[14] N. Auerbach and Amir Klein, Phys. Rev. C 28, 2075 (1983).

[15] N. Auerbach, Phys. Rev. Lett. 49, 913 (1982).

[16] S. A. Wood, Los Alamos National Laboratory Report No. LA-9932-T, 1984 (unpul)lished).

[17] J. L. Matthews, Proceedings of the LAMPF Workshop on Pion Double Charge Exchange, [Los Alamos National Laboratory Report No. LA-10550-C, 1985 (u11)ub)lished)].

[18] A. H. Wapstra, G. Audi, and R. Hockstra, At. and Nuc. Data Tables, 39, 281 (1988). 40, 9 (1988). 
Table 1: Target compositions, areal densities, and angles of measured data points.

\begin{tabular}{lccl}
\hline \hline & $\begin{array}{c}\text { Isotopic } \\
\text { purity } \\
(\%)\end{array}$ & $\begin{array}{c}\text { Areal } \\
\text { density } \\
\left(\mathrm{g} / \mathrm{cm}^{2}\right)\end{array}$ & $\begin{array}{l}\text { Scattering } \\
\text { Angles } \\
(\text { lab })\end{array}$ \\
\hline${ }^{13} \mathrm{C}$ & 90.0 & 0.329 & $5^{\circ}$ \\
${ }^{27} \mathrm{Al}$ & 100.0 & 1.713 & $5^{\circ}$ \\
${ }^{40} \mathrm{Ca}$ & 96.9 & 2.38 & $5^{\circ}, 12^{\circ}, 19^{\circ}$ \\
${ }^{56} \mathrm{Fe}$ & 91.8 & 2.44 & $5^{\circ}$ \\
${ }^{59} \mathrm{Co}$ & 100 & 1.079 & $5^{\circ}$ \\
${ }^{93} \mathrm{Nb}$ & 100 & 3.428 & $5^{\circ}$ \\
\hline \hline
\end{tabular}

Table 2: Double dipole $Q$ values and excitation energies for the $\left(\pi^{-}, \pi^{+}\right)$reaction extracted from the present study compared with previous data from the $\left(\pi^{+}, \pi^{-}\right)$reaction (Rof. [2]) measured on the same target nuclei.

\begin{tabular}{cccccccc}
\hline \hline \multirow{2}{*}{ Target } & \multicolumn{3}{c}{$\left(\pi^{+}, \pi^{-}\right)$} & & \multicolumn{3}{c}{$\left(\pi^{-}, \pi^{+}\right)$} \\
\cline { 2 - 4 } \cline { 7 - 8 } & $\begin{array}{c}Q_{g s}{ }^{\mathbf{a}} \\
(\mathrm{MeV})\end{array}$ & $\begin{array}{c}Q_{D D} \\
(\mathrm{MeV})\end{array}$ & $\begin{array}{c}E_{x}(D D) \\
(\mathrm{MeV})\end{array}$ & & $\begin{array}{c}Q_{g s}{ }^{\mathbf{a}} \\
(\mathrm{MeV})\end{array}$ & $\begin{array}{c}Q_{D D} \\
(\mathrm{MeV})\end{array}$ & $\begin{array}{c}E_{x}(D D) \\
(\mathrm{MeV})\end{array}$ \\
\hline${ }^{13} \mathrm{C}$ & -18.96 & $-46.5 \pm 2.0$ & $27.5 \pm 2.0$ & & -32.84 & $-49.5 \pm 0.5$ & $16.7 \pm 0.5$ \\
${ }^{27} \mathrm{Al}$ & -15.42 & $-49.1 \pm 0.5$ & $33.7 \pm 0.5$ & & -12.57 & $-36.2 \pm 0.3$ & $23.6 \pm 0.3$ \\
${ }^{40} \mathrm{Ca}$ & -24.86 & $-54.0 \pm 0.5$ & $29.1 \pm 0.5$ & & -0.83 & $-31.1 \pm 0.3$ & $30.3 \pm 0.3$ \\
${ }^{56} \mathrm{Fe}$ & -5.68 & $-54.4 \pm 0.6$ & $48.7 \pm 0.6$ & & -6.33 & $-30.0 \pm 0.5$ & $23.7 \pm 0.5$ \\
${ }^{59} \mathrm{Co}$ & -4.85 & $-48.6 \pm 0.8$ & $43.8 \pm 0.8$ & & -7.77 & $-35.0 \pm 0.5$ & $27.2 \pm 0.5$ \\
${ }^{93} \mathrm{Nb}$ & -2.58 & $-49.9 \pm 0.8$ & $47.3 \pm 0.8$ & & -3.99 & $-24.9 \pm 1.0$ & $20.9 \pm 1.0$ \\
\hline \hline
\end{tabular}

a Values from the 1986 mass table, Ref [18]. 


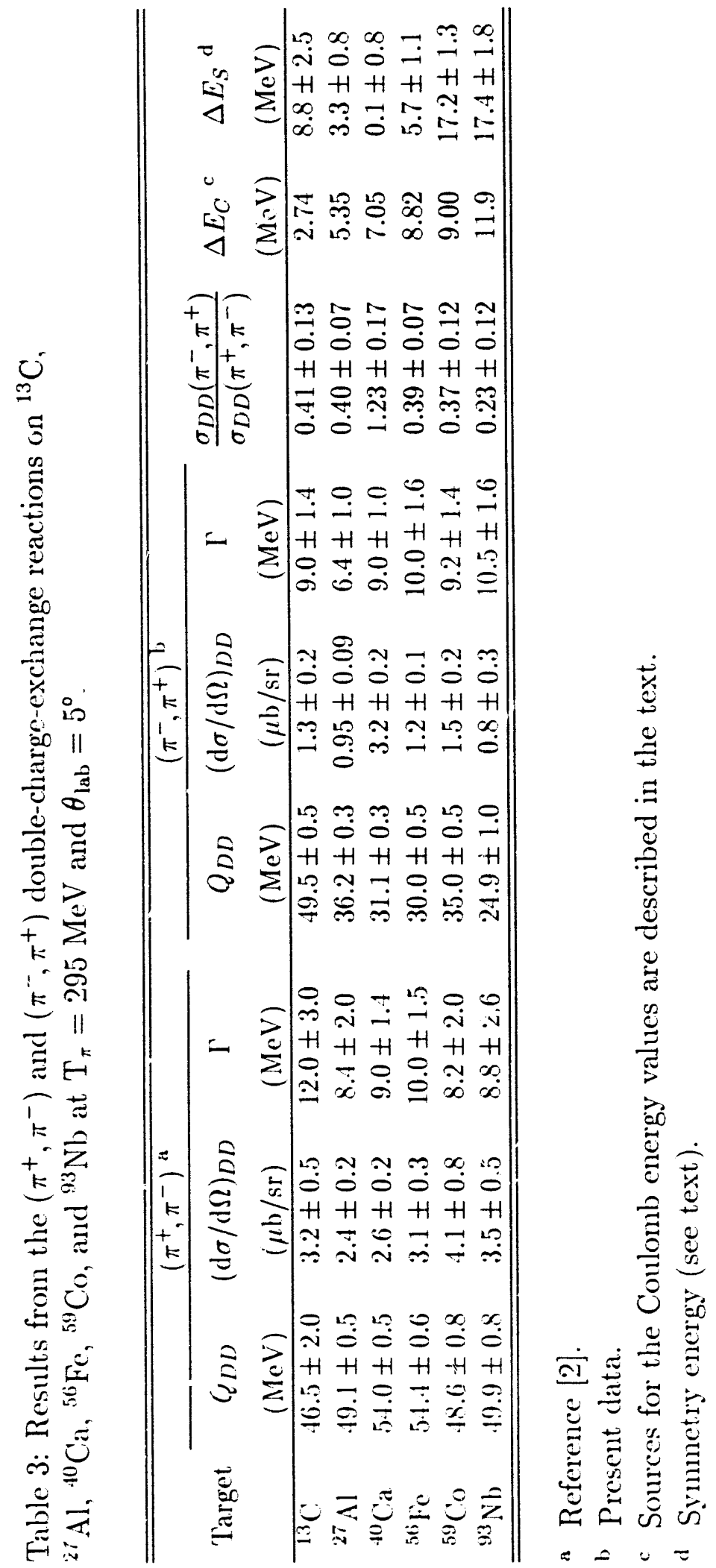


Table 4: Comparison between the observed Pauli blocking for the double-dipole in DCX, the single-dipole in SCX, and theoretical estimates for exciting the isovector giant dipole.

\begin{tabular}{cccc}
\hline \hline Target & $R\left(\mathrm{GDR}^{2}\right)^{\mathrm{a}}$ & $R(\mathrm{GDR})^{\mathrm{b}}$ & $R(\mathrm{GDR})_{\mathrm{th}}{ }^{\mathrm{c}}$ \\
\hline${ }^{13} \mathrm{C}$ & $0.41 \pm 0.13$ & - & - \\
${ }^{27} \mathrm{Al}$ & $0.40 \pm 0.07$ & - & - \\
${ }^{40} \mathrm{Ca}$ & $1.23 \pm 0.17$ & $1.18 \pm 0.33$ & 1.37 \\
${ }^{56} \mathrm{Fe}$ & $0.39 \pm 0.07$ & - & - \\
${ }^{59} \mathrm{Co}$ & $0.37 \pm 0.12$ & $0.45 \pm 0.20$ & $0.45^{\mathrm{d}}$ \\
${ }^{93} \mathrm{Nb}$ & $0.23 \pm 0.12$ & $0.43 \pm 0.20$ & $0.52^{\mathrm{e}}$ \\
\hline \hline
\end{tabular}

a $R\left(\mathrm{GDR}^{2}\right)=\sigma_{D D}\left(\pi^{-}, \pi^{+}\right) / \sigma_{D D}\left(\pi^{+}, \pi^{-}\right)$. The double dipole cross sections are taken from Table III.

b $R(\mathrm{GDR})=\sigma_{\mathrm{GDR}}\left(\pi^{-}, \pi^{0}\right) / \sigma_{\mathrm{GDR}}\left(\pi^{+}, \pi^{0}\right)$. GDR peak cross sections from single charge exchange (Ref. [12]). For ${ }^{59} \mathrm{Co}$ and ${ }^{93} \mathrm{Nb}$ we give the ratios measured for ${ }^{60} \mathrm{Ni}$ and ${ }^{90} \mathrm{Zr}$ respectively.

c Ref. [14].

d Theoretical value for ${ }^{60} \mathrm{Ni}$ (Ref. [14]).

e Theoretical value for ${ }^{90} \mathrm{Zr}$ (Ref. [14]). 


\section{Publications}

1. M. K. Jones, D. Dehnhard, S. K. Nanda, S. M. Sterbenz, C. L. Morris, M. Plum, J. D. Zumbro, Ahmed Hussein, D. S. Oakley, M. J. Smithson, A. L. Williams, J. McDonald, M. A. Bryan, A. H. Fuentes, M. Lynker, D. Crockett, M. A. Machuca, S. Mordechai, and C. Fred Moore, " ${ }^{4} \mathrm{He}\left(\pi, \pi^{\prime} \mathrm{p}\right)^{3} \mathrm{H}$ reaction: quasifree and resonance scattering," Physical Review C 42, R807-R810 (1990).

2. J. M. O'Donnell, H. T. Fortune, J. D. Silk, C. L. Morris, J. D. Zumbro, S. Mordechai, C. Fred Moore, and S. H. Yoo, "The ${ }^{138} \mathrm{Ce}(\mathrm{T}=13)$ double isobaric analog state studied by pion induced double charge exchange," Physical Review C submitted (1990).

3. R. D. Ransome, V. R. Cupps, S. Dawson, R. W. Fergerson, A. Green, C. L. Morris, J. A. McGill, J. R. Comfort, B. G. Ritchie, J. R. Tinsley, J. D. Zumbro, R. A. Loveman, P. C. Gugelot, D. L. Watson, and C. Fred Moore, "Systematics of pion absorption on ${ }^{6} \mathrm{Li}, "$ Physical Review C 42, 1500-1518 (1990).

4. P. A. Seidl, M. A. Bryan, M. Burlein, G. R. Burleson, Kalvir S. Dhuga, H. T. Fortune, R. Gilman, S. J. Greene, M. A. Machuca, C. Fred Moore, S. Mordechai, C. L. Mo is, D. S. Oakley, M. A. Plum, G. Rai, M. J. Smithson, Z. F. Wang, D. L. Watson, and J. D. Zumbro, "Pion double charge exchange on $T=2$ nuclei in the $\Delta_{3 / 2,3 / 2}$ resonance region," Physical Review C 42, 1929-1934 (1990).

5. S. Mordechai and C. Fred Moore, "Observation of double giant dipole resonances in pion double charge exchange," Proceedings of the Second International Conference on Medium and High-Energy Nuclear Physics, Taiwan, Republic of China, May 14-18, 361-376 (1990).

6. G. S. Blanpied, J. Hernandez, C. S. Mishra, W. K. Mize, C. S. Whisnant, B. G. Ritchie, C. L. Morris, S. J. Seestrom-Morris, C. Fred Moore, P. A. Seidl, R. A. Lindgren, B. H. Wildenthal, and R. Gilman, "Pion elastic and inelastic scattering. from ${ }^{24} \mathrm{Mg}$ and ${ }^{25} \mathrm{Mg}$," Physical Review C 41, 1625-1636 (1990).

7. Yi-Fen Yen, B. Brinkmöller, D. Dehnhard, S. M. Sterbenz, Yi-Ju Yu, Brian Berman, G. R. Burleson, K. Cranston, A. Klein, G. S. Kyle, R. Alarcon, T. Averett, J. R. Comfort, J. J. Gögen, B. G. Ritchie, J. R. Tinsley, M. Barlett, G. W. Hoffmann, K. Johnson, C. F. Moore, M. Purcell, H. Ward, A. Williams, J. A. Faucett, S. J. Greene, J. J. Jarmer, J. A. McGill, C. L. Morris, S. Penttilä, N. Tanaka, H. T. Fortune, E. Insko, R. Ivie, J. M. O'Donnell, D. Smith, M. A. Khandaker, S. Chakravarti, "Asymmetry measurement of pion elastic scattering from polarized ${ }^{13} \mathrm{C}$ in the energy" region of the $P_{33}$ resonance," Physical Review Letters 66, 1959-1962 (1991).

8. D. L. Watson, R. Gilman, C. L. Morris, J. D. Zumbro, S. Mordechai, M. Burlein, J. M. O'Donnell, H. T. Fortune, A. Kotwal, J. Puttz, J. D. Silk, J. S. McDonald, C. 
Fred Moore, A. L. Williams, and S. Y. Yoo, "Pion-induced double charge exchange on ${ }^{12} \mathrm{C},{ }^{24} \mathrm{Mg},{ }^{32} \mathrm{~S}$, and ${ }^{40} \mathrm{Ca}, "$ Physical Review C 43, 1318-1320 (1991).

9. A. L. Williams, J. A. McGill, C. L. Morris, G. R. Burleson, J. A. Faucett, D. S. Oakley, M. Burlein, H. T. Fortune, J. M. O'Donnell, G. P. Kahrimanis, and C. Fred Moore, "Pion double charge exchange on ${ }^{42,44,48} \mathrm{Ca}$ for $300 \leq T_{\pi} \leq 550 \mathrm{McV}$," Physical Review C 43, 766-770 (1991).

10. S. Mordechai, C. L. Morris, J. M. O'Donnell, M. A. Kagarlis, D. Fink, H. T. Fortune, D. L. Watson, R. Gilman, H. Ward, A. Williams, Sung Hoon Yoo, and C. Fred Moore, "Analog of the $T_{>}$giant dipole resonance in light nuclei," Physical Review C 43, 11111115 (1991).

11. S. Mordechai, H. Ward, K. Johnson, G. Kahrimanis, David Saunders, C. Fred Moore, J. M. O'Donnell, M. A. Kagarlis, D. Smith, H. T. Fortune, C. L. Morris, "Comparison of the double giant-dipole states observed in $\left(\pi^{-}, \pi^{+}\right)$and $\left(\pi^{+}, \pi^{-}\right)$reactions on ${ }^{40} \mathrm{Ca}$ and ${ }^{27}$ Al," Physical Review C 43, R1509-R1512 (1991).

12. S. Mordechai and C. Fred Moore, "Giant resonances in single and double charge exchange," Proceedings of the International Workshop on Pions in Nuclei, Peniscola, Spain (1991).

13. C. L. Morris, R. D. Ransome, B. G. Ritchie, J. D. Zumbro, D. L. Watson, and C. Fred Moore, "Evidence for physics beyond the quasi-deuteron model in pion absorption," Proceedings of the International Workshop on Pions in Nuclei, Peniscola, Spain (1991).

14. S. Mordechai and C. Fred Moore, "Double giant resonances in atomic nuclei," NATURE 352, 393-397 (1991).

15. H. T. Fortune, S. Mordechai, C. Fred Moore, et al., "Pion double charge exchange on ${ }^{24} \mathrm{Mg}$ at $165 \mathrm{MeV}$," in preparation.

16. M. K. Jones, R. D. Raisome, V. R. Cupps, R. W. Fergerson, C. L. Morris, J. A. McGill, J. D. Zumbro, J. R. Comfort, B. G. Ritchie, J. R. Tinsley, P. C. Gugelot, C. Fred Moore, "Pion absorption above the $\Delta(1232)$ resonance," Physical Revieu Letters, submitted July (1991).

17. N. M. Hintz, X. H. Yang, M. Gazzaly, S. J. Seestrom-Morris, C. L. Morris, D. C. Cook, A. M. Mack, J. S. McDonald, D. S. Oakley, C. F. Moore, M. Lynker, J. D. Zumbro, "Determination of neutron and proton multipole matrix elements in ${ }^{208} \mathrm{~Pb}$ from $\pi^{-}$and $\pi^{+}$scattering at $180 \mathrm{MeV}$," Physical Review C, submitted June (1091).

18. R. D. Ransome, C. L. Morris, V. R. Cupps, R. W. Fergerson, J. A. McGill, A. Grecn, S. Dawson, D. L. Watson, J. D. Zumbro, B. G. Ritchie, J. R. Comfort, J. R. Tinsley, R. A. Loveman, P. C. Gugelot, C. Fred Moore, "Pion absorption in heavy nuclei," Physical Review C, submitted June (1991). 
19. D. S. Oakley, R. J. Peterson, S. J. Seestrom, C. L. Morris, M. A. Plum, J. D. Zumbro, A. L. Williams, M. A. Bryan, J. W. McDonald, C. Fred Moore, "Energy dependence of pion inelastic scattering from ${ }^{208} \mathrm{~Pb}, "$ Physical Review C, submitted June (1991).

20. A. L. Williams, K. W. Johnson, G. P. Kahrimanis, H. Ward, C. Fred Moore, J. A. McGill, C. L. Morris, G. R. Burleson, J. A. Faucett, M. Rawool-Sullivan, D. S. Oakley, M. Burlein, H. T. Fortune, E. Insko, R. Ivie, J. M. O'Donnell, D. Smith, "Mass dependence of high-energy pion double charge exchange," Physical Revieu $C$, submitted August (1991).

21. C. Fred Moore, K. Johnson, G. P. Kahrimanis, James McDonald, M. Snell, H. J. Ward, Sung Hoon Yoo, C. L. Morris, S. Mordechai, M. Burlein, N. Claytor, H. T. Fortune, R. Ivie, G. B. Liu, J. M. O'Donnell, D. Smith, N. Auerbach, and D. Robson, "Angular distributions for the double isobaric analog and a $\mathrm{T}_{<}$state at high excitation in pion double charge exchange on ${ }^{93} \mathrm{Nb}, "$ Physical Review C, in press (1991).

22. H. Ward, J. Johnson, C. Whitley, C. Fred Moore, J. Applegate, J. Beck, B. G. Ritchie, S. Mordechai, M. Rawool-Sullivan, and D. Watson, "Excitation mechanism for the analog of the anti-analog in pion DCX in heavy nuclei," in preparation (1991).

23. H. Ward, K. Johnson, G. Kahrimanis, D. Saunders, C. Fred Moore, S. Mordechai, C. L. Morris, J. M. O'Donnell, N. Auerbach, M. A. Kagarlis, H. T. Fortune, and D. Smith, "The double giant dipole resonance in the $\left(\pi^{-}, \pi^{+}\right)$reaction," Physical Review $C$ submitted (1991).

24. S. Mordechai, C. Fred Moore al., "The lowest three $0^{+}$states in ${ }^{12} \mathrm{O}$ from pion double charge exchange on ${ }^{12} \mathrm{C} "$, in preparation (1991).

25. E. Gülmez, D. L. Adams, S. Beedoe, M. Bleszynski, J. Bystricky, V. Ghazikhanian, G. Igo, T. Jaroszewicz, A. G. Ling, M. Nasser, M. W. McNaughton, S. Penttilä, G. Glass, G. Kahrimanis, W. F. Kielhorn, I. H. McNaughton, P. J. Riley, S. Sen, D. Wolf, "Elastic scattering of N, L, and S-Type polarized $794 \mathrm{MeV}$ protons from an $\mathrm{ND}_{3}$ target polarized in the S-L Plane," (submitted to Physical Review C, April 1991.)

26. Herb Ward, C. Fred Moore, P. Susie Maloney, and Jimmy D. Martin, "Probabilistic Neural Nets in Analysis of Magnetic Spectrometer Data", (submitted to NATURE, Aug 1991).

27. J. L. Langenbrunner, M. K. Jones, D. Dehnhard, S. K. Nanda, S. M. Sterbenz, C. L. Morris, M. Plum, J. D. Zumbro, A. Hussein, D. S. Oakley, M. Smithson, A. Williams, J. McDonald, M. Bryan, A. Fuentes, M. Lynker, D. Crockett, M. Machuca, S. Mordechai, and C. Fred Moore, "Coincidence Measurement of ${ }^{4} \mathrm{He}\left(\pi, \pi^{\prime} p\right)$ and ${ }^{4} \mathrm{H}\left(\pi, p i^{\prime} t\right), "$ Physical Review $C$, submitted (1991). 


\section{Abstracts}

1. H. J. Ward, K. Johnson, G. P. Kahrimanis, D. Saunders, C. Fred Moore, Shaul Mordechai, C. L. Morris, J. M. O'Donnell, H. T. Fortune, M. A. Kargarlis, D. Smith, "Observation of the double giant dipole resonance in $\left(\pi^{-}, \pi^{+}\right)$double charge exchange," Bull. Am. Phys. Soc. 36, 99 (1991).

2. S. Mordechai, C. L. Morris, J. M. O'Donnell, H. T. Fortune, M. A. Kagarlis, D. Smith, H. J. Ward, A. Williams, Sung Hoon Yoo, C. Fred Moore, and D. L. Watson, "Observation of the $T_{>}$giant dipole resonance in light nuclei," Bull. Am. Phys. Soc. 36, 99 (1991).

3. K. Johnson, M. Purcell, H. Ward, A. Williams, S. Worm, B. Brinkmöller, D. Dehnhard, J. Langenbrunner, M. Palarczyk, C. Reidel, Y. Yen, B. Burleson, M. Ghossain, A. Klein, G. Kyle, S. Mukhopadhyay, M. Wang, R. Ivie, M. Kagarlis, S. Loe, M. Mackenzie, J. O'Donnell, D. Smith, M. Khandaker, M. Khayat, C. Allgower, J. Comfort, S. Greene, J. Jarmer, S. Penttila, and N. Tanaka, "Pion elastic and inelastic scattering on polarized ${ }^{13}$ C," Bull. Am. Phys. Soc. 36, 99 (1991).

4. J. L. McDonald, M. A. Bryan, C. Fred Moore, J. W. McDonald, Thomas E. Cowan, C. L. Bennett, M. Clark, R. H. Howell, R. R. Rohatgi, D. H. G. Schneider, J. Schweple, J. Fajans, "Positron-electron spectroscopy in heavy-ion collisions," Bull. Am. Phys. Soc. 36, 98 (1991).

5. C. L. Morris, John McGill, Ronald Ransome, Mark Jones, David Clayton, Junal Pujara, Barry Ritchie, Ian Brown, Paul Campbell, Doug Watson, and C. Fred Moore, "Excitation function of partial cross sections for pion absorption on ${ }^{12} \mathrm{C}$," Bull. Am. Phys. Soc. 36, 1299 (1991).

6. B. G. Ritchie, J. M. Applegate, T. D. Averett, J. Beck, D. S. Oakley, C. Kormanyos, R. J. Peterson, J. A. McGill, C. L. Morris, C. F. Moore, M. Snell, and H. Ward, "50 and $65 \mathrm{MeV} \pi^{ \pm}$scattering from ${ }^{12} \mathrm{C}$ with the Scruncher," Bull. Am. Phys. Soc. 36, 1299 (1991).

7. S. Mordechai and C. Fred Moore, "Double resonance excitations in pion double charge exchange", Notre Dame Workshop on Giant Resonances and Related Phenomena, Notre Dame, Indiana, Oct. 21-23 (1991).

8. Yi-Fen Yen, B. Brinkmoller, D. Dehnhard, S. M. Sterbenz, Yi-Ju Yu, B. Berman, G. R. Burleson, K. Cranston, A. Klein, G. S. Kyle, R. Alarcon, T. Averett, J. R. Comfort, J. Georgen, B. Ritchie, J. R. Tinsley, G. W. Hoffmann, K. Johnson, M. Purcell, H. Ward, A. Williams, J. A. Faucett, S. J. Greene, J. Jarmer, J. A. McGill, C. L. Morris, S. Penttila, N. Tanaka, H. T. Fortune, E. Insko, R. Ivie, J. M. O'Donncll, D. Smith, S. Hoibraten, M. Khandaker, S. Chakravarti, "Pion elastic scattering from 
polarized ${ }^{13} \mathrm{C}$ in the energy region of the $[3,3]$ resonance", submitted to the 9th International Symposium on High Energy Physics, Bonn, October 10-15, 1990 and the PARIS 90 Conference, Paris, July 9-13, 1990, Colloque de. Physique, Colloque C6, supplement qu n²2, Tome 51, 15 Novembre 1990.

\section{Invited Talks}

\section{Since September 1990}

1. S. Mordechai and C. Fred Moore, "Higher-energy pion double charge exchange", Annual Meeting LAMPF Users Group, Pilac Working Group, Los Alamos National I aboratory, Los Alamos, New Mexico (1990).

2. S. Mordechai, "Two-step giant dipole excitations in nuclei", Annual Mecting of the Israel Physical Society, Beer Sheva, Israel (1991).

3. S. Mordechai and C. Fred Moore, "Giant resonances in single and double chinge exchange", International Workshop on Pions in Nuclei, Peniscola, Spain (1991).

4. S. Mordechai and C. Fred Moore, "Giant resonances", Pion Double Charge Exchange Workshop, DCX Working Group, Los Alamos National Laboratory, Los Alamos, New Mexico (1991)

5. S. Mordechai and C. Fred Moore, "Double resonance excitations in pion double chinge exchange", Notre Dame, Indiana ("1991).

\section{Proposal Defenses}

Since August 1990

1. "Measuring neutron-neutron scattering length and effective range using the ${ }^{2} \mathrm{H}\left(\pi^{-}, 211\right) \gamma$ reaction", LAMPF Proposal 1182.

2. "Energy dependence of double resonances in pion DCX", LAMPF Proposal 1209.

3. "Study of the mechanism for DCX to excite analogs of anti-analogs in heavy nuclci", LAMPF Proposal 1210.

4. " $\left(\pi, \pi^{\prime} X\right)$ coincidence measurement on and below the $\Delta_{3,3}$ resonance", LAMPF Proposal 1211 .

5. "6 $\operatorname{Li}\left(\pi, \pi^{\prime} X\right)$ coincidence measurements near the $\Delta_{3,3}$ resonance", LAMPF Experiment 1216 .

6. "Accurate determination of the $T_{>}$giant dipole state in light $T=1 / 2$ nuclei using pion DCX", LAMPF Proposal 1217. 
7. "Elastic scattering of $\pi^{+}$and $\pi^{-}$from ${ }^{4} \mathrm{He}$ and ${ }^{3} \mathrm{He}$ above the $\Delta_{3,3}$ resonance", LAMPF Proposal 1219.

8. "A study of the pion-nucleus isovector spin-flip mechanism at low energies using "B", LAMPF Proposal 1226.

9. "The two-proton component of the pion absorption reaction on nuclei at low encrgies", LAMPF Proposal 1227.

10. "Feasibility of the direct production of pionic atoms at LAMPF", LAMPF Proposal 1239.

11. "Isospin structure of the giant dipole resonance in pion single charge exchange", LAMPF Proposal 1241.

12. "The double giant dipole resonance in deformed nuclei", LAMPF Proposal 1242.

13. "Giant resonance excitations by low energy pion scattering", LAMPF Proposal 1253.

\section{Approved New Research Proposals}

Since August 1990

1. "Measuring neutron-neutron scattering length and effective range using the ${ }^{2} \mathrm{H}\left(\pi^{-}, 2 n\right) \gamma$ reaction", LAMPF Experiment \#1182.

2. "Energy dependence of double resonances in pion DCX", LAMPF Experiment \#1209.

3. "Study of the mechanism for DCX to excite analogs of anti-analogs in heavy nuclei", LAMPF Experiment \#1210.

4. "6 $\operatorname{Li}\left(\pi, \pi^{\prime} X\right)$ coincidence measurements near the $\Delta_{3,3}$ resonance", LAMPF Experiment \#1216.

5. "Accurate determination of the $T_{>}$giant dipole state in light $T=1 / 2$ nuclei using pion DCX", LAMPF Experiment \#1217.

6. "Elastic scattering of $\pi^{+}$and $\pi^{-}$from ${ }^{4} \mathrm{He}$ and ${ }^{3} \mathrm{He}$ above the $\Delta_{3,3}$ resonance", LAMPF Experiment \#1219.

7. "A study of the pion-nucleus isovector spin-flip mechanism at low energies using ${ }^{10} \mathrm{~B}$ ", LAMPF Experiment \#1226.

8. "The two-proton component of the pion absorption reaction on nuclei at low cnergies", LAMPF Experiment \#1227.

9. "Feasibility of the direct production of pionic atoms at LAMPF", LAMPF Expcriment \#1239. 
10. "Isospin structure of the giant dipole resonance in pion single charge exchange", LAMPF Experiment \#1241.

11. "Giant resonance excitations by low energy pion scattering", LAMPF Experinent \#1253.

\section{Personnel}

Since September 1990

William. T. Buttler

Steve Hamilton

John Johnson

Iievin Johnson

George P. Kahrimanis

James L. McDonald

Joseph W. McDonald

C. Fred Moore

Shaul Mordechai

Arturo Morosoff

Dave P. Saunders

Mike Snell

Herbert J. Ward

Charles Whitley

Allen Williams graduate student

graduate student

graduate student

graduate student

graduate student

graduate student

graduate student

faculty

faculty (Ben Gurion University of the Negev)

graduate student

graduate student (Air Force Academy)

graduate student (Air Force Academy)

graduate student

graduate student

graduate student (University of Pennsylvania)

Institutions in parentheses indicate either current employment or a permanent place of employment. 

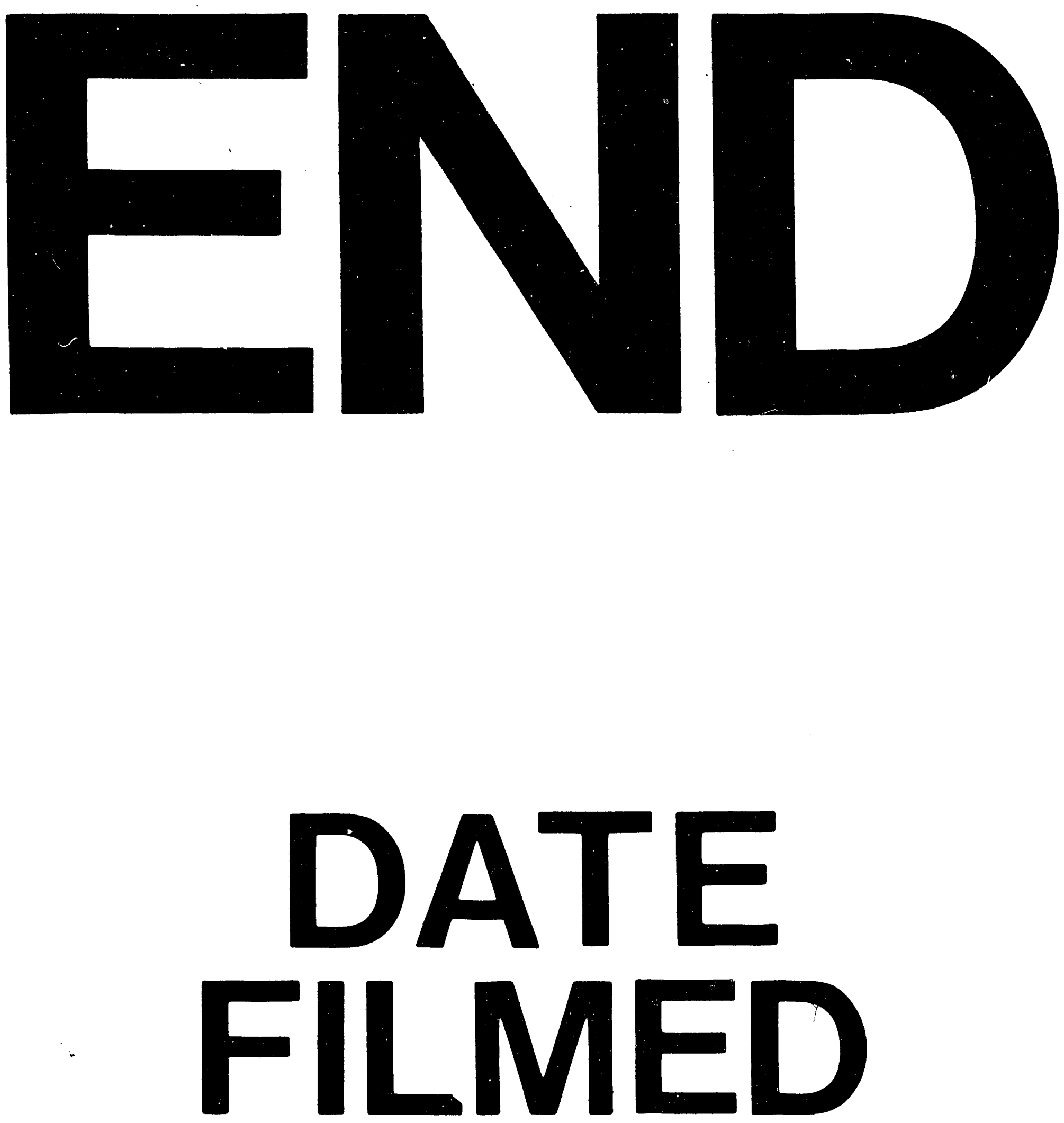

7

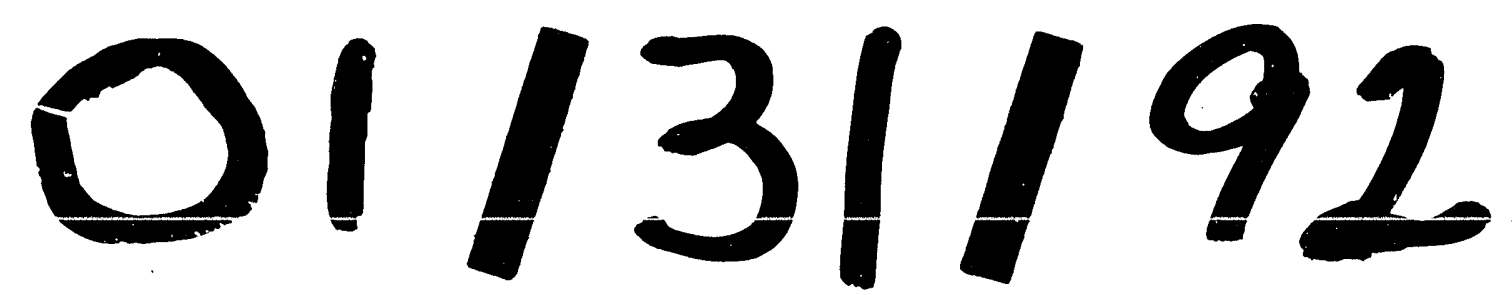


